\title{
VALUES OF THE EULER $\phi$-FUNCTION NOT DIVISIBLE BY A GIVEN ODD PRIME, AND THE DISTRIBUTION OF EULER-KRONECKER CONSTANTS FOR CYCLOTOMIC FIELDS
}

\author{
KEVIN FORD, FLORIAN LUCA, AND PIETER MOREE
}

\begin{abstract}
Let $\phi$ denote Euler's phi function. For a fixed odd prime $q$ we investigate the first and second order terms of the asymptotic series expansion for the number of $n \leqslant x$ such that $q \nmid \phi(n)$. Part of the analysis involves a careful study of the Euler-Kronecker constants for cyclotomic fields. In particular, we show that the Hardy-Littlewood conjecture about counts of prime $k$-tuples and a conjecture of Ihara about the distribution of these EulerKronecker constants cannot be both true.
\end{abstract}

\section{INTRODUCTION}

Let $B(x)$ denote the counting function of integers $n \leqslant x$ that can be written as the sum of two squares. In 1908, Landau 25] proved the asymptotic formula

$$
B(x) \sim \frac{K x}{\sqrt{\log x}}
$$

for a certain positive constant $K$. Landau's proof is based on the analytic theory of Dirichlet $L$-functions, which come into play because a number $n$ is the sum of two squares if and only if each prime $p \mid n$ with $p \equiv 3(\bmod 4)$ divides $n$ with an even exponent. The next year, Landau ([26]; see also [27, §176-183]) found a general asymptotic for the number of integers $n \leqslant x$ which are divisible by no prime $p \in S$, where $S$ is any set of reduced residue classes modulo a fixed, but arbitrary, positive integer $q$. In the case where $q$ is an odd prime and $S=\{1(\bmod q)\}$, let $\mathcal{A}_{q}(x)$ be the counting function of such $n$.

Let $\phi$ denote Euler's phi function. For fixed odd prime $q$, let

$$
\mathcal{E}_{q}(x)=|\{n \leqslant x: q \nmid \phi(n)\}| .
$$

Since $q \nmid \phi(n)$ if and only if $q^{2} \nmid n$ and $p \nmid n$ for all primes $p \equiv 1(\bmod q)$ ), it follows that $\mathcal{E}_{q}(x)=\mathcal{A}_{q}(x)-\mathcal{A}_{q}\left(x / q^{2}\right)$. Landau's theorem immediately implies that

$$
\mathcal{E}_{q}(x) \sim \frac{e_{0}(q) x}{(\log x)^{\frac{1}{q-1}}}
$$

for some positive constant $e_{0}(q)$.

Received by the editor January 17, 2012 and, in revised form, June 20, 2012 and August 22, 2012 .

2010 Mathematics Subject Classification. Primary 11N37, 11Y60.

The first author was supported in part by National Science Foundation grants DMS-0555367 and DMS-0901339.

The second author was supported in part by Grants SEP-CONACyT 79685 and PAPIIT 100508 . 
A standard application of the Selberg-Delange method (e.g., [6, Theorem B]) gives an asymptotic expansion

$$
\mathcal{E}_{q}(x)=\frac{x}{(\log x)^{1 /(q-1)}}\left(e_{0}(q)+\frac{e_{1}(q)}{\log x}+\cdots+\frac{e_{k}(q)}{\log ^{k} x}+O_{k}\left(\frac{1}{\log ^{k+1} x}\right)\right),
$$

with $e_{j}(q)$ being certain constants depending on $q$ and $k \geqslant 1$ an arbitrary natural number. One of the main topics of this paper is the behavior of the second order terms $e_{1}(q)$ from (1.3).

To place our problem in historical context, recall that Gauss's approximation $\operatorname{li}(x)=\int_{2}^{x} d t / \log t$ is a much better estimate of $\pi(x)$, the number of primes up to $x$, than is $x / \log x$. Possibly inspired by this fact, in his first letter (16 Jan. 1913) to Hardy, Ramanujan claimed that, for every $r \geqslant 1$,

$$
B(x)=K \int_{2}^{x} \frac{d t}{\sqrt{\log t}}+O\left(\frac{x}{\log ^{r} x}\right) .
$$

However, Shanks [47] showed that (1.4) is false for every $r>3 / 2$. On the other hand he showed that the first term in (1.4) yields a closer approximation to $B(x)$ than does $K x / \sqrt{\log x}$. Similarly, in an unpublished manuscript, possibly included with his final letter (12 Jan. 1920) to Hardy, Ramanujan discussed congruence properties of $\tau(n)$, the coefficient of $q^{n}$ in $q \prod_{k=1}^{\infty}\left(1-q^{k}\right)^{24}$, and $p(n)$, the partition function (see [1] or [3]). For a finite set of special primes $q$ and positive constants $\delta_{q}$, Ramanujan claimed that "it can be shown by transcendental methods that

$$
\sum_{\substack{n \leqslant x \\ q \nmid \tau(n)}} 1=C_{q} \int_{2}^{x} \frac{d t}{(\log t)^{\delta_{q}}}+O\left(\frac{x}{\log ^{r} x}\right),
$$

where $r$ is an positive number". Although asymptotically correct (as shown by Rankin and Rushforth), the third author [37] showed that all claims of the form (1.5) are false for every $r>1+\delta_{q}$.

It is natural to ask which of the following two approximations is asymptotically closer to $\mathcal{E}_{q}(x)$, the Landau approximation

$$
\mathcal{L}_{q}(x)=\frac{e_{0}(q) x}{\log ^{1 /(q-1)} x}
$$

or the Ramanujan approximation

$$
\mathcal{R}_{q}(x)=e_{0}(q) \int_{2}^{x} \frac{d t}{\log ^{1 /(q-1)} t} .
$$

Integration by parts gives

$$
\mathcal{R}_{q}(x)=\frac{e_{0}(q)}{(\log x)^{\frac{1}{q-1}}}\left(1+\frac{1}{(q-1) \log x}+O\left(\frac{1}{\log ^{2} x}\right)\right),
$$

and it follows that if $(q-1) e_{1}(q) / e_{0}(q)>\frac{1}{2}$, then there exists $x_{0}$ such that

$$
\left|\mathcal{E}_{q}(x)-\mathcal{R}_{q}(x)\right|<\left|\mathcal{E}_{q}(x)-\mathcal{L}_{q}(x)\right|, \forall x \geqslant x_{0} .
$$

If (1.6) holds, we say that the Ramanujan approximation is asymptotically closer than the Landau approximation. 
Theorem 1. Let $q$ be an odd prime. For $q \leqslant 67$ the Ramanujan approximation $\mathcal{R}_{q}(x)$ is asymptotically closer than the Landau approximation $\mathcal{L}_{q}(x)$ for $\mathcal{E}_{q}(x)$, and for all remaining primes the Landau approximation is asymptotically closer. That is, $(q-1) e_{1}(q) / e_{0}(q)>\frac{1}{2}$ precisely when $q \leqslant 67$.

Whereas before only a finite number of "Landau versus Ramanujan" comparison problems were settled, Theorem 1 extends this to an infinite number. The following result reveals, in fact, that neither $\mathcal{L}_{q}(x)$ nor $\mathcal{R}_{q}(x)$ capture the second term of the expansion (1.3). Throughout this paper, by ERH we mean that all nontrivial zeros of the Dirichlet $L$-functions for characters modulo $q$ lie on the critical line $\Re s=\frac{1}{2}$.

Theorem 2. We have

$\frac{e_{1}(q)}{e_{0}(q)}=\frac{1-\gamma}{q-1}+ \begin{cases}O\left(\frac{\log ^{2} q}{q^{3 / 2}}\right), & \text { unconditionally with an effective constant, } \\ O \varepsilon\left(\frac{1}{q^{2}-\varepsilon}\right), & \forall \varepsilon>0, \text { unconditionally with an ineffective constant } \\ O\left(\frac{\log ^{2} q}{q^{2}}\right), & \text { if there are no exceptional zeros for } q \\ O\left(\frac{(\log q) \log \log q}{q^{2}}\right), & \text { on ERH for L-functions modulo } q .\end{cases}$

Here $\gamma=0.57721566 \ldots$ is Euler's constant, and in this paper, an exceptional zero is a real number $\beta>1-1 /(9.645908801 \log q)$, that is, a zero of $L\left(s, \chi_{q}\right)$, with $\chi_{q}$ being the real, nonprincipal character modulo $q$.

McCurley [34, Theorem 1.1] showed that for each $q$, the region

$$
\Re s \geqslant 1-\frac{1}{9.645908801 \log \max (q, q|\Im s|, 10)}
$$

contains at most one zero of $\prod_{\chi} \bmod q L(s, \chi)$, and if the zero exists, it is real, simple and a zero of $L\left(s, \chi_{q}\right)$.

The remainder of the introduction is organized as follows. Subsection 1.1 presents the necessary analytic theory to understand $e_{0}(q)$. In subsection 1.2, we express the ratio $e_{1}(q) / e_{0}(q)$ in terms of two additional quantities $S(q)$ and $\gamma_{q}$, (defined in (1.11) and (1.12), respectively) and which are intesting to study in their own right. We also state a theorem about the behavior of $S(q)$. Subsection 1.3 gives some general background on $\gamma_{q}$ (called an Euler-Kronecker constant), and in subsection 1.4 we present several theorems and conjectures about $\gamma_{q}$.

A paper by Spearman and Williams [48] inspired us to study $\mathcal{E}_{q}(x)$. In a rather roundabout way they obtained the asymptotic (1.2) (but not (1.3)) and gave an expression for $e_{0}(q)$ involving invariants of cyclotomic fields. We point out in the next subsection that by using the Dedekind zeta function of a cyclotomic field, one can rederive their expression (1.10) for $e_{0}(q)$ more directly.

1.1. The first order term in (1.3). The basis of (1.3) is an analysis of the Dirichlet series generating function for $n$ with $q \nmid \phi(n)$, namely

$$
h_{q}(s)=\left(1+q^{-s}\right) \prod_{\substack{p \neq q \\ p \neq 1(\bmod q)}}\left(1-p^{-s}\right)^{-1}=\left(1-q^{-2 s}\right) \zeta(s) \prod_{p \equiv 1(\bmod q)}\left(1-p^{-s}\right),
$$

where $\zeta(s)$ is the Riemann zeta function. Roughly speaking, the Selberg-Delange method provides an asymptotic expansion for $\sum_{n \leqslant x} a_{n}$ in decreasing powers of $\log x$ provided that the Dirichlet series $\sum_{n=1}^{\infty} a_{n} n^{s}$ behaves like $\zeta(s)^{z}$ for some fixed complex number $z$. If $a_{n}$ is multiplicative, this means that $a_{p}$ has an average value 
$z$ over primes $p$. In our case, $z=\frac{q-2}{q-1}$ by the prime number theorem for arithmetic progressions. We observe that the primes $p \equiv 1(\bmod q)$ are precisely those primes which split completely in $K(q):=\mathbb{Q}\left(e^{2 \pi i / q}\right)$ and thus $\zeta_{K(q)}(s)$, the Dedekind zeta function of $K(q)$, comes into play. We prove the following in Section 2 .

Proposition 1. Let $q$ be an odd prime. Then

$$
h_{q}(s)=\frac{\left(1-q^{-2 s}\right) \zeta(s)}{\left[C(q, s)\left(1-q^{-s}\right) \zeta_{K(q)}(s)\right]^{\frac{1}{q-1}}},
$$

where

$$
C(q, s)=\prod_{\substack{p \neq q \\ f_{p} \geqslant 2}}\left(1-\frac{1}{p^{s f_{p}}}\right)^{\frac{q-1}{f_{p}}}
$$

and $f_{p}=\operatorname{ord}_{q} p$ (the least positive $f$ with $\left.p^{f} \equiv 1(\bmod q)\right)$. Furthermore,

$$
e_{0}(q)=\frac{1-q^{-2}}{\Gamma\left(\frac{q-2}{q-1}\right)\left(C(q)\left(1-\frac{1}{q}\right) \alpha_{K(q)}\right)^{\frac{1}{q-1}}}, \quad \alpha_{K(q)}=\operatorname{Res}_{s=1} \zeta_{K(q)}(s) .
$$

The main result in Spearman and Williams [48, is the asymptotic (1.2) with $e_{0}(q)$ expressed in terms of the parameters of $K(q)$; namely,

$$
e_{0}(q)=\frac{(q+1)(q-1)^{\frac{q-2}{q-1}} \Gamma\left(\frac{1}{q-1}\right) \sin \left(\frac{\pi}{q-1}\right)}{2^{\frac{q-3}{2(q-1)}} q^{\frac{3(q-2)}{2(q-1)}} \pi^{\frac{3}{2}}(h(K(q)) R(K(q)) C(q))^{\frac{1}{q-1}}},
$$

where $h(K(q))$ denotes the class number of $K(q)$ and $R(K(q))$ is its regulator. Spearman and Williams gave a rather involved description of $C(q)$; see Section 2.2, Making use of the Euler product for $\zeta_{K(q)}(s)$, we will show that $C(q)=C(q, 1)$. We have, for example, $C(3)=\prod_{p \equiv 2(\bmod 3)}\left(1-1 / p^{2}\right)($ this is Lemma 3.1 of [48]). Our argument also gives a very short proof of an estimate of a product from [48] (inequality (2.9) below). By using that $\Gamma\left(\frac{1}{q-1}\right) \Gamma\left(\frac{q-2}{q-1}\right)=\frac{\pi}{\sin (\pi /(q-1))}$ and formula (2.1) for $\alpha_{K(q)}$ below, it is seen that the $e_{0}(q)$ as given in Proposition 1 matches the formula (1.10).

1.2. The second order term in (1.3). Our argument for Theorems 1 and 2 proceed by first relating the $e_{1}(q) / e_{0}(q)$ to two additional quantities,

$$
S(q)=\frac{1}{q-1} \frac{C^{\prime}(q, 1)}{C(q, 1)}=\sum_{p \neq q, f_{p} \geqslant 2} \frac{\log p}{p^{f_{p}}-1}
$$

and the Euler-Kronecker constant

$$
\gamma_{q}=\gamma_{K(q)}=\lim _{s \rightarrow 1}\left(\frac{\zeta_{K(q)}(s)}{\alpha_{K(q)}}-\frac{1}{s-1}\right) .
$$

Proposition 2. We have

$$
(q-1) \frac{e_{1}(q)}{e_{0}(q)}=1-\gamma+\frac{(3-q) \log q}{(q-1)^{2}(q+1)}+S(q)+\frac{\gamma_{q}}{q-1} .
$$

In Section 4, we prove the following upper estimates for $S(q)$ : 


\section{Theorem 3.}

(a) We have $S(q) \leqslant 45 / q$ for all $q$;

(b) Let $\epsilon>0$ be fixed. The inequality $S(q)<\epsilon / q$ holds for $(1+o(1)) \pi(x)$ primes $q \leqslant x$.

The analysis used to prove Theorem 3 depends on estimates for linear forms in logarithms to deal with the summands with $p$ and $f_{p}$ both small.

As we will see, $\gamma_{q}$ is typically around $\log q$ and hence Theorem 3 allows us to deduce that $\gamma_{q}$ has a larger influence on the ratio $e_{1}(q) / e_{0}(q)$ than does $S(q)$. The Euler-Kronecker constant (or invariant) can be defined for any number field. Some history and basics will be recalled in the next section.

1.3. Euler-Kronecker constants for number fields. For a general number field $K$ we have, for $\Re(s)>1$, the Dedekind zeta function

$$
\zeta_{K}(s)=\sum_{\mathfrak{a}} \frac{1}{N \mathfrak{a}^{s}}=\prod_{\mathfrak{p}} \frac{1}{1-N \mathfrak{p}^{-s}} .
$$

Here, a runs over nonzero ideals in $\mathcal{O}_{K}$, the ring of integers of $K, \mathfrak{p}$ runs over the prime ideals in $\mathcal{O}_{K}$ and $N \mathfrak{a}$ is the norm of $\mathfrak{a}$. It is known that $\zeta_{K}(s)$ can be analytically continued to $\mathbb{C}-\{1\}$, and that at $s=1$ it has a simple pole with residue $\alpha_{K}$, where [17, Theorem 61$]$

$$
\alpha_{K}=\frac{2^{r_{1}}(2 \pi)^{r_{2}} h(K) R(K)}{w(K) \sqrt{|d(K)|}},
$$

where $K$ has $r_{1}$ (resp. $2 r_{2}$ ) real (resp. complex) embeddings, class number $h(K)$, regulator $R(K), w(K)$ roots of unity, and discriminant $d(K)$. The Dedekind zeta function $\zeta_{K}(s)$ has a Laurent expansion

$$
\zeta_{K}(s)=\frac{\alpha_{K}}{s-1}+c_{0}(K)+c_{1}(K)(s-1)+c_{2}(K)(s-1)^{2}+\cdots .
$$

The ratio $\gamma_{K}=c_{0}(K) / \alpha_{K}$ is called the Euler-Kronecker constant of $K$ (in particular, $\gamma_{\mathbb{Q}}=\gamma$ is Euler's constant). This terminology originates with Ihara [22]. In the older literature (for references up to 1984 see, e.g., Deninger [7]) the focus was on determining $c_{0}(K)$. As Tsfasman [50] points out, $\gamma_{K}$ is of order $\log \sqrt{|d(K)|}$, whereas $\alpha_{K}$ may happen to be exponential in $\log \sqrt{|d(K)|}$.

The case where $K$ is quadratic has a long history. Since $\zeta_{\mathbb{Q}(\sqrt{D})}(s)=\zeta(s) L\left(s, \chi_{D}\right)$, with $\chi_{D}=(D / n)$ the Kronecker symbol, we obtain

$$
\gamma_{\mathbb{Q}(\sqrt{D})}=\gamma+\frac{L^{\prime}\left(1, \chi_{D}\right)}{L\left(1, \chi_{D}\right)}
$$

by partial integration and using that $L\left(1, \chi_{D}\right) \neq 0$. In the case when $K$ is imaginary quadratic the well-known Kronecker limit formula expresses $\gamma_{K}$ in terms of special values of the Dedekind $\eta$-function (see e.g. Section 2.2 in [22]). An alternative expression involves a sum of logarithms of the Gamma function at rational values. Equating both expressions the Chowla-Selberg formula is obtained. Deninger [7] worked out the analogue of the latter formula for real quadratic fields.

Numerical example.

$$
\gamma_{\mathbb{Q}(i)}=\gamma+\frac{L^{\prime}\left(1, \chi_{-4}\right)}{L\left(1, \chi_{-4}\right)}=\log \left(\frac{\xi^{2}}{2} e^{2 \gamma}\right) \approx 0.82282525, \text { where } \xi=\sqrt{\frac{2}{\pi}} \Gamma\left(\frac{3}{4}\right)^{2} .
$$


(The number $\xi$ is also the arithmetic-geometric-mean (AGM) of 1 and $\sqrt{2}$.)

Put

$$
\tilde{\zeta}_{K}(s)=s(1-s)\left(\frac{\sqrt{|d(K)|}}{2^{r_{2}} \pi^{[K: \mathbb{Q}] / 2}}\right)^{s} \Gamma\left(\frac{s}{2}\right)^{r_{1}} \Gamma(s)^{r_{2}} \zeta_{K}(s) .
$$

Then it is known that the functional equation $\tilde{\zeta}_{K}(s)=\tilde{\zeta}_{K}(1-s)$ holds. Since $\tilde{\zeta}_{K}(s)$ is entire of order 1 , one has the Hadamard product factorization

$$
\tilde{\zeta}_{K}(s)=\tilde{\zeta}_{K}(0) e^{\beta_{K} s} \prod_{\rho}\left(1-\frac{s}{\rho}\right) e^{s / \rho},
$$

with some complex number $\beta_{K}$. Hashimoto et al. [20] (cf. Ihara [22, pp. 416-421]) showed that

$$
-\beta_{K}=\sum_{\rho} \frac{1}{\rho}=\gamma_{K}-\left(r_{1}+r_{2}\right) \log 2+\frac{1}{2} \log |d(K)|-\frac{[K: \mathbb{Q}]}{2}(\gamma+\log \pi)+1,
$$

where the sum is over the zeros of $\zeta_{K}(s)$ in the critical strip. By specializing this to the case $K(q)$, we obtain

$$
\sum_{\zeta_{K(q)}(\rho)=0} \frac{1}{\rho}=\gamma_{q}-(q-1)(\log 2+\gamma)+\frac{1}{2}(q-2) \log q-\frac{(q-1)}{2} \log \pi .
$$

Since, at least conjecturally, $\gamma_{q}$ has normal order $\log q$ (see Theorem 6 below), this quantity seems to "measure" a subtle effect in the distribution of the zeros.

Prime ideals of small norm in the ring of integers of $K$ have a large influence on $\gamma_{K}$ as the following result (see, e.g., 20]) shows:

$$
\gamma_{K}=\lim _{x \rightarrow \infty}\left(\log x-\sum_{N \mathfrak{p} \leqslant x} \frac{\log N \mathfrak{p}}{N \mathfrak{p}-1}\right) .
$$

As we shall see in the next subsection, in the special case $K=K(q), \gamma_{q}$ is heavily influenced by small primes which are congruent to 1 modulo $q$.

1.4. Euler-Kronecker constants for cyclotomic fields. In Section 3 we study the distribution of $\gamma_{q}$ as $q$ runs through the primes. In particular, we will give explicit estimates for these constants needed for proving Theorems 1 and 2 .

In [22, Ihara remarks that it seems very likely that always $\gamma_{q}>0$ (this was checked numerically for $q \leqslant 8000$ by Mahoro Shimura, assuming ERH). Ihara observed that $\gamma_{K}$ can be conspicuously negative and that this occurs when $K$ has many primes having small norm (cf. (1.16)). However, in the case of $K(q)$ the smallest norm is $q$ and therefore is rather large as $q$ increases.

Using a new, fast algorithm (requiring computation of $L(1, \chi)$ for all characters modulo $q$; see formula (2.6) below), we performed a search for small values of $\gamma_{q}$. The details of the algorithm and computation are described later in Section 3 . One negative value was found at $q=964477901$. We discuss later in this subsection the reason why this $q$, and conjecturally infinitely many others, have negative EulerKronecker constants.

Theorem 4. For $q=964477901$, we have

$$
\gamma_{q}=-0.18237 \ldots
$$

In 22, Ihara also proved, under the assumption of ERH, the one-sided bound $\gamma_{q} \leqslant(2+o(1)) \log q$. In 23], Ihara made the following stronger conjecture. 
Conjecture I (Ihara). For any $\epsilon>0$, if $q$ is sufficiently large, then

$$
\left(\frac{1}{2}-\epsilon\right) \log q<\gamma_{q}<\left(\frac{3}{2}+\epsilon\right) \log q .
$$

We will show, assuming the Hardy-Littlewood conjectures for counts of prime $k$-tuples, that the lower bound in Ihara's conjecture is false and, even more, that $\gamma_{q}$ is infinitely often negative. In 1904, Dickson [8] posed a wide generalization of the twin prime conjecture that is now known as the "prime $k$-tuples conjecture". It states that whenever a set of linear forms $a_{i} n+b_{i}\left(1 \leqslant i \leqslant k, a_{i} \geqslant 1, b_{i} \in\right.$ $\mathbb{Z}$ ) have no fixed prime factor (there is no prime $p$ that divides $\prod_{i}\left(a_{i} n+b_{i}\right)$ for all $n$ ), then for infinitely many $n$, all of the numbers $a_{i} n+b_{i}$ are prime. This expresses a kind of local-to-global principle for prime values of linear forms, but is has not been proven for any $k$-tuple of forms with $k \geqslant 2$. Later, Hardy and Littlewood [19] conjectured an asymptotic formula for the number of such $n$. There have been extensive numerical studies of prime $k$-tuples, in particular, in the case $a_{1}=\cdots=a_{k}=1$, providing evidence for these conjectures (e.g. [13, 14]).

In connection with $\gamma_{q}$, we need to understand special sets of forms. We say that a set $\left\{a_{1}, \ldots, a_{k}\right\}$ of positive integers is an admissible set if the collection of forms $n$ and $a_{i} n+1(1 \leqslant i \leqslant k)$ have no fixed prime factor. We need the following weak form of the Hardy-Littlewood conjecture:

Conjecture HL. Suppose $\mathcal{A}=\left\{a_{1}, \ldots, a_{k}\right\}$ is an admissible set. The number of primes $n \leqslant x$ for which the numbers $a_{i} n+1$ are all prime is $\gg_{\mathcal{A}} x(\log x)^{-k-1}$.

Theorem 5. Assume Conjecture HL. Then

$$
\liminf _{q \rightarrow \infty} \frac{\gamma_{q}}{\log q}=-\infty .
$$

The basis of our theorem is the following formula for $\gamma_{q}$; cf. (1.16).

Proposition 3. We have

$$
\begin{aligned}
\gamma_{q} & =-\frac{\log q}{q-1}+\lim _{x \rightarrow \infty}\left[\log x-(q-1) \sum_{\substack{n \leqslant x \\
n \equiv 1(\bmod q)}} \frac{\Lambda(n)}{n}\right] \\
& =-\frac{\log q}{q-1}-(q-1) S(q)+\lim _{x \rightarrow \infty}\left[\log x-(q-1) \sum_{\substack{p \leqslant x \\
p \equiv 1(\bmod q)}} \frac{\log p}{p-1}\right] .
\end{aligned}
$$

The Euler-Kronecker constant $\gamma_{q}$ may also be easily expressed in terms of Dirichlet $L$-functions at $s=1$; see (2.6) below in $\$ 2.1$

It is expected that the primes $p \equiv 1(\bmod q)$ behave very regularly for $p>q^{1+\varepsilon}$ (arbitrary fixed $\varepsilon>0$ ). It is irregularities in the distribution of the $p \leqslant q^{1+\varepsilon}$ which provide the variation in the values of $\gamma_{q}$.

Put $a(1)=0$ and inductively define $a(n)$ to be the smallest integer exceeding $a(n-1)$ such that, for every prime $r$, the set $\{a(i)(\bmod r): 1 \leqslant i \leqslant n\}$ has at most $r-1$ elements (using the Chinese remainder theorem it is easily seen that the sequence is infinite). Given the prime $k$-tuples conjecture an equivalent statement is that $a(n)$ is minimal such that there are infinitely many primes $q$ with $q+a(i)$ prime for $1 \leqslant i \leqslant n$. We have $\{a(i)\}_{i=1}^{\infty}=\{0,2,6,8,12,18,20,26,30,32, \ldots\}$. This is sequence A135311 in the OEIS [43] and is called "the greedy sequence of prime 
offsets". Given the prime $k$-tuples conjecture another equivalent statement is that $a(n)$ is minimal such that $a(1)=0$ and there are infinitely many primes $q$ with $a(i) q+1$ prime for $2 \leqslant i \leqslant n, n \geqslant 2$. Define $i_{0}$ to be the smallest integer satisfying

$$
\sum_{i=2}^{i_{0}} \frac{1}{a(i)}>2
$$

A computer calculation gives $i_{0}=2089$ and $a\left(i_{0}\right)=18932$.

Proposition 4. Suppose that the number of primes $q$ such that $a(i) q+1$ is a prime for $2 \leqslant i \leqslant 2089$ is $\gg x /(\log x)^{2090}$. Then $\gamma_{q}<0$ for $\gg x /(\log x)^{2090}$ primes $q \leqslant x$.

We note here that when $q=964477901$, then $a q+1$ is prime for

$$
a \in\{2,6,8,12,18,20,26,30,36,56, \ldots\} .
$$

The strongest unconditional result about the distribution of primes in arithmetic progressions, the Bombieri-Vinogradov theorem, implies that the primes $p \equiv 1(\bmod q)$ with $p>q^{2}(\log q)^{A}$ are well-distributed for most $q$. The ElliottHalberstam conjecture [9] goes further: Let $\pi(x ; q, 1)$ denote the number of primes $p \leqslant x$ such that $p \equiv 1(\bmod q)$. For convenience, write

$$
E(q ; x)=\pi(x ; q, 1)-\frac{\operatorname{li}(x)}{\phi(q)} .
$$

Conjecture EH (Elliott-Halberstam). For every $\varepsilon>0$ and $A>0$,

$$
\sum_{q \leqslant x^{1-\varepsilon}}|E(q ; x)| \ll_{A, \varepsilon} \frac{x}{(\log x)^{A}} .
$$

Theorem 6. (i) Assume Conjecture EH. For every $\varepsilon>0$, the bounds

$$
1-\varepsilon<\frac{\gamma_{q}}{\log q}<1+\varepsilon
$$

hold for almost all primes $q$ (that is, the number of exceptional $q \leqslant x$ is o $(\pi(x)$ ) as $x \rightarrow \infty)$.

(ii) Assume Conjectures $H L$ and EH. Then the set $\left\{\gamma_{q} / \log q: q\right.$ prime $\}$ is dense in $(-\infty, 1]$.

If, as widely believed, $E(x ; q)$ is small for all $q \leqslant x^{1-\varepsilon}$, we may make a stronger conclusion.

Conjecture 1. The set of limit points of $\left\{\gamma_{q} / \log q: q\right.$ prime $\}$ is $(-\infty, 1]$.

To determine the maximal order of $-\gamma_{q}$, one needs to assume a version of Conjecture HL with the implied constant in the $\gg$-symbol explicitly depending on $\left\{a_{1}, \ldots, a_{k}\right\}$. The heuristic argument in [15, Proposition 5 and $\left.\S 9\right]$ suggests that perhaps

$$
\liminf \frac{\gamma_{q}}{(\log q)(\log \log \log q)}=-1
$$

Our conditional results about $\gamma_{q}$ are proved using standard methods of analytic number theory, and are very similar to the conditional bounds given by Granville in [15] for the class number ratio $h_{q}^{-}:=h\left(\mathbb{Q}\left(e^{2 \pi i / q}\right)\right) / h(\mathbb{Q}(\cos 2 \pi / q))$. Kummer in 1851 conjectured that, as $q \rightarrow \infty$, one has

$$
h_{q}^{-} \sim 2 q\left(\frac{q}{4 \pi^{2}}\right)^{(q-1) / 4} .
$$


This conjecture is the analog of the conjecture that $\gamma_{q} \sim \log q$ as $q \rightarrow \infty$. We will make use of several results from [15].

Our Theorem 5 is reminiscent of a theorem of Hensley and Richards [21, who showed the incompatibility of the prime $k$-tuples conjecture and a conjecture of Hardy and Littlewood about primes in short intervals.

Coming back to the connection between $\gamma_{q}$ and zeros of $\zeta_{K(q)}(s)$ (cf. (1.15)), assuming ERH Ihara [23] defined

$$
c(q):=\left(\sum_{\rho} \frac{q^{\rho-1 / 2}}{\rho(1-\rho)}\right) / \sum_{\rho} \frac{1}{\rho(1-\rho)}=\left(\sum_{\rho} \frac{\cos (\tau \log q)}{\frac{1}{4}+\tau^{2}}\right) / \sum_{\rho} \frac{1}{\frac{1}{4}+\tau^{2}},
$$

where $\rho=1 / 2+i \tau$ runs over all nontrivial zeros of $\zeta_{K(q)}(s)$. We have $|c(q)| \leqslant 1$ and

$$
\left(\int_{\infty}^{\infty} \frac{\cos (t \log q)}{\frac{1}{4}+t^{2}} d t\right) /\left(\int_{-\infty}^{\infty} \frac{d t}{\frac{1}{4}+t^{2}}\right)=\frac{1}{\sqrt{q}}
$$

Thus, assuming that the distribution of $\tau$ modulo $2 \pi / \log q$ for small $|\tau|$ is rather uniform, we would maybe expect that $\sqrt{q} c(q)$ approximates 1 closely. Ihara 23 , Proposition 3] showed that under ERH we have

$$
\frac{\gamma_{q}}{\log q}=\frac{3}{2}+(\sqrt{q} c(q)-1)+O\left(\frac{1}{\log q}\right) .
$$

However, assuming ERH and Conjecture HL, it follows from this and Theorem 5 that

$$
\liminf _{q \rightarrow \infty} \sqrt{q} c(q)=-\infty .
$$

Furthermore, assuming Conjecture EH, Theorem 6(i) and (1.17) lead to the conjecture that the normal order of $\sqrt{q} c(q)$ is $1 / 2$.

1.5. The Euler-Kronecker constant for multiplicative sets. A set $S$ of positive integers is said to be multiplicative if for every pair $m$ and $n$ of coprime positive integers we have that $m n$ is in $S$ iff both $m$ and $n$ are in $S$. In other words, $S$ is a multiplicative set if and only if the indicator function $f_{S}$ of $S$ is a multipicative function.

Example 1. The set of positive integers that can be written as a sum of two squares.

Example 2. The set $S_{q}:=\{n \geqslant 1: q \nmid \phi(n)\}$.

The Dirichlet series $L_{S}(s):=\sum_{n=1, n \in S}^{\infty} n^{-s}$ converges for $\Re s>1$. If $L_{S}(s)$ has a simple pole at $s=1$ with residue $\delta>0$ and if

$$
\gamma_{S}:=\lim _{s \rightarrow 1+0}\left(\frac{L_{S}^{\prime}(s)}{L_{S}(s)}+\frac{\delta}{s-1}\right)
$$

exists, we say that $S$ has Euler-Kronecker constant $\gamma_{S}$. If we suppose that there exist $\delta, \rho>0$ such that

$$
\sum_{p \leqslant x, p \in S} 1=\delta \pi(x)+O\left(\frac{x}{\log ^{2+\rho} x}\right),
$$

then it can be shown that $\gamma_{S}$ exists. In this terminology some of our results take a nicer form, e.g., in Theorem 2 we now have $\gamma_{S_{q}}=\gamma+O_{\epsilon}\left(q^{\epsilon-1}\right)$ (with an ineffective constant). For details and further results the reader is referred to Moree [40. 
Finally, we like to point out that this paper is a very much reworked version of an earlier preprint (2006) by the third author [39. In it a proof of Theorem 1 on ERH is given. From the perspective of computational number theory, this proof is far easier and less computation intensive than the one that does not assume ERH given here.

\section{AnAlytic theory}

2.1. Propositions 1 and 2, We recall some facts from the theory of cyclotomic fields needed for our proofs. For a nice introduction to cyclotomic fields, see [52]. The following result (see e.g. [42, Theorem 4.16]) describes the splitting of primes in the ring of integers of a cyclotomic field.

Lemma 1 (cyclotomic reciprocity law). Let $K=\mathbb{Q}\left(e^{2 \pi i / m}\right)$. If the prime $p$ does not divide $m$ and $f=\operatorname{ord}_{m} p$, then the principal ideal $p \mathcal{O}_{K}=\mathfrak{p}_{1} \cdots \mathfrak{p}_{g}$ with $g=\phi(m) / f$, and all $\mathfrak{p}_{\mathfrak{i}}$ 's are distinct and of degree $f$.

However, if $p$ divides $m, m=p^{a} m_{1}$ with $p \nmid m_{1}$ and $f=\operatorname{ord}_{m_{1}} p$, then $p \mathcal{O}_{k}=\left(\mathfrak{p}_{1} \cdots \mathfrak{p}_{g}\right)^{e}$ with $e=\phi\left(p^{a}\right), g=\phi\left(m_{1}\right) / f$, and all $\mathfrak{p}_{\mathfrak{i}}$ 's are distinct and of degree $f$.

In case $K=K(q)$, we have $r_{1}=0,2 r_{2}=q-1, w(K)=2 q$ (as $K$ contains exactly $\left\{ \pm 1, \pm \omega, \pm \omega^{2}, \ldots, \pm \omega^{q-1}\right\}$ as roots of unity, with $\left.\omega=e^{2 \pi i /(q-1)}\right)$ and furthermore $D(K)=(-1)^{q(q-1) / 2} q^{q-2}$, and thus we obtain from (1.13) that

$$
\alpha_{K(q)}=\operatorname{Res}_{s=1} \zeta_{K}(s)=2^{\frac{q-3}{2}} q^{-\frac{q}{2}} \pi^{\frac{q-1}{2}} h(K) R(K) .
$$

For cyclotomic fields $K$ the Euler product for $\zeta_{K}(s)$ can be written explicitly using the "cyclotomic reciprocity law". We find that

$$
\begin{aligned}
\zeta_{K(q)}(s) & =\left(1-\frac{1}{q^{s}}\right)^{-1} \prod_{p \neq q}\left(1-\frac{1}{p^{s f_{p}}}\right)^{\frac{1-q}{f_{p}}} \\
& =\left(1-\frac{1}{q^{s}}\right)^{-1} C(q, s)^{-1} \prod_{p \equiv 1(\bmod q)}\left(1-\frac{1}{p^{s}}\right)^{1-q} .
\end{aligned}
$$

It is also well known (see, e.g., [17, Theorem 65]) that

$$
\zeta_{K(q)}(s)=\zeta(s) \prod_{\chi \neq \chi_{0}} L(s, \chi)
$$

where the product is over characters $\chi$ modulo $q$, with $\chi_{0}$ being the principal character, and $L(s, \chi)$ the Dirichlet $L$-function associated with $\chi$.

Proof of Proposition 1, First, (1.8) follows by combining (1.7) and (2.2). By (2.3),

$$
h_{q}(s)=\left(1+q^{-s}\right)\left(\zeta(s)\left(1-q^{-s}\right)\right)^{\frac{q-2}{q-1}} C(q, s)^{-\frac{1}{q-1}} \prod_{\chi \neq \chi_{0}} L(s, \chi)^{-\frac{1}{q-1}} .
$$

For $\chi \neq \chi_{0}, L(s, \chi)$ is analytic and nonzero at $s=1$. Therefore, $f(s)=h_{q}(s)(s-1)^{(q-2) /(q-1)} s^{-1}$ is analytic in a neighborhood of $s=1$ and has a power series expansion there. Moreover, $\prod_{\chi \neq \chi_{0}} L(s, \chi)$ has no zeros in the region $\Re s \geqslant 1-a_{q}(\log (|\Im s|+2))^{-1}$ for some positive $a_{q}$. Therefore, $h_{q}(s) / s$ has an expansion around the point $s=1$ of the form

$$
\frac{h_{q}(s)}{s}=\frac{1}{(s-1)^{(q-2) /(q-1)}}\left(c_{0}(q)+c_{1}(q)(s-1)+\cdots+c_{k}(q)(s-1)^{k}+\cdots\right) .
$$


To apply the Selberg-Delange method, we also need a mild growth condition on $h_{q}(s) \zeta(s)^{-\frac{q-2}{q-1}}$. The function $C(q, s)$ is analytic for $\Re s>\frac{1}{2}$, and uniformly bounded in the half-plane $\Re s \geqslant \frac{3}{4}$. For $\sigma \geqslant 1-a_{q}(2 \log (|t|+2))^{-1}$,

$$
\left|\prod_{\chi \neq \chi_{0}} L(\sigma+i t)\right|^{-1} \ll_{q}(\log (|t|+2))^{q-2} .
$$

By [49, §II.5, Theorem 3], the asymptotic expansion (1.3) holds with the coefficients satisfying $e_{j}(q)=c_{j}(q) / \Gamma\left(\frac{q-2}{q-1}-j\right)$.

Proof of Proposition 2, By Proposition 1 and the functional equation

$$
\Gamma(z)=(z-1) \Gamma(z-1),
$$

we have

$$
\begin{aligned}
\frac{e_{1}(q)}{e_{0}(q)} & =-\frac{1}{q-1} \frac{c_{1}(q)}{c_{0}(q)}=-\frac{f^{\prime}(1)}{(q-1) f(1)} \\
& =\frac{1}{q-1}\left(1-\lim _{s \rightarrow 1^{+}}\left(\frac{1-\frac{1}{q-1}}{s-1}+\frac{h_{q}^{\prime}(s)}{h_{q}(s)}\right)\right) .
\end{aligned}
$$

By the Laurent expansion $\zeta(s)=(s-1)^{-1}+\gamma+O(|s-1|)$, we have

$$
\frac{\zeta^{\prime}(s)}{\zeta(s)}=-\frac{1}{s-1}+\gamma+O(|s-1|) \quad(|s-1| \leqslant 1) .
$$

Hence, by logarithmic differentiation of (2.4),

$\lim _{s \rightarrow 1^{+}} \frac{1-\frac{1}{q-1}}{s-1}+\frac{h_{q}^{\prime}(s)}{h_{q}(s)}=-\frac{\log q}{q+1}+\frac{(q-2) \log q}{(q-1)^{2}}+\frac{q-2}{q-1} \gamma-S(q)-\frac{1}{q-1} \sum_{\chi \neq \chi_{0}} \frac{L^{\prime}(1, \chi)}{L(1, \chi)}$.

By (1.14), (2.5) and logarithmic differentiation of (2.3), we have

$$
\gamma_{q}=\gamma+\sum_{\chi \neq \chi_{0}} \frac{L^{\prime}(1, \chi)}{L(1, \chi)}
$$

By combining the various formulas the proof is completed.

2.2. The constant $C(q)$. Spearman and Williams put, for a generator $\chi_{q}$ of the group of characters modulo $q$,

$$
C(q)=\prod_{r=1}^{q-2} \prod_{\chi_{g}(p)=\omega^{r}}\left(1-\frac{1}{p^{(q-1) /(r, q-1)}}\right)^{(r, q-1)} .
$$

From this definition it is not a priori clear that $C(q)$ is independent of the choice of $\chi_{g}$. However, Spearman and Williams show that this is so.

Proposition 5. We have $C(q)=C(q, 1)$.

Proof. We claim that if $\chi_{g}(p)=\omega^{r}$, then $f_{p}=(q-1) /(r, q-1)$. We have $1=\chi_{g}\left(p^{f_{p}}\right)=\omega^{r f_{p}}$. It follows that $(q-1) \mid r f_{p}$ and thus $q_{r}=(q-1) /(r, q-1)$ must be a divisor of $f_{p}$. On the other hand, since $\chi_{g}(a)=1$ if and only if $a=1$, 
it follows from $\omega^{r q_{r}}=\chi_{g}\left(p^{q_{r}}\right)=1$ and $q_{r} \mid f_{p}$, that $f_{p}=q_{r}$. Thus, we can rewrite (2.7) as

$$
C(q)=\prod_{r=1}^{q-2} \prod_{\chi_{g}(p)=\omega^{r}}\left(1-\frac{1}{p^{f_{p}}}\right)^{q-\frac{1}{f_{p}}} .
$$

Note that $p \neq q$ and $f_{p} \geqslant 2$ iff $\chi_{g}(p)=\omega^{r}$ for some $1 \leqslant r \leqslant q-2$. This observation in combination with the absolute convergence of the double product (2.8), then shows that $C(q)=C(q, 1)$.

Remark. Proposition 5 says that $1 / C(q)$ is the contribution at $s=1$ of the primes $p \neq q, p \not \equiv 1(\bmod q)$ to the Euler product (2.2) of $K(q)$.

2.3. On Mertens' theorem for arithmetic progressions. A crucial ingredient in the paper of Spearman and Williams is the asymptotic estimate [48, Proposition 6.3] that

$$
\prod_{\substack{p \leqslant x \\ p \equiv 1(\bmod q)}}\left(1-\frac{1}{p}\right)=\left(\frac{q e^{-\gamma}}{(q-1) \alpha_{K(q)} C(q) \log x}\right)^{\frac{1}{q-1}}\left(1+O_{q}\left(\frac{1}{\log x}\right)\right) .
$$

An alternative, much shorter proof of the estimate (2.9) can be obtained by invoking Mertens' theorem for algebraic number fields.

Lemma 2. Let $\alpha_{K}$ denote the residue of $\zeta_{K}(s)$ at $s=1$. Then

$$
\prod_{N \mathfrak{p} \leqslant x}\left(1-\frac{1}{N \mathfrak{p}}\right)=\frac{e^{-\gamma}}{\alpha_{K} \log x}\left(1+O_{K}\left(\frac{1}{\log x}\right)\right),
$$

where the product is over the prime ideals $\mathfrak{p}$ in $\mathcal{O}_{K}$ having norm $\leqslant x$.

Proof. Similar to that of the usual Mertens' theorem (see e.g. Rosen [44] or Lebacque [32]).

Proof of estimate (2.9). We invoke Lemma 2 with $K=K(q)$ and work out the product over the prime ideals more explicitly using the cyclotomic reciprocity law, Lemma 1. One finds, for $x \geqslant q$, that it equals

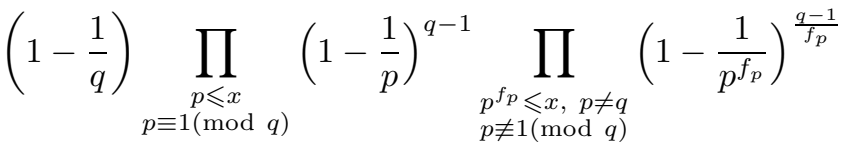

$$
\begin{aligned}
& =\left(1+O_{q}\left(\frac{1}{\sqrt{x}}\right)\right)\left(1-\frac{1}{q}\right) C(q) \prod_{\substack{p \leqslant x \\
p \equiv 1(\bmod q)}}\left(1-\frac{1}{p}\right)^{q-1},
\end{aligned}
$$

where we used that for $k \geqslant 2$,

$$
\prod_{p^{k}>x}\left(1-p^{-k}\right)^{-1}=1+O\left(\sum_{n^{k}>x} n^{-k}\right)=1+O\left(x^{1 / k-1}\right) .
$$

Thus, by invoking Lemma 2, we deduce (2.9).

For recent work on this theme, the reader is referred to the papers by Languasco and Zaccagnini 28 31. 


\section{Estimates for the Euler-Kronecker constants $\gamma_{q}$}

\subsection{Unconditional bounds for $\gamma_{q}$.}

Proof of Proposition 3. Apply (2.6), the orthogonality of characters, and the relation (e.g. [27, §55] or [36, §6.2, Exercise 4])

$$
\sum_{n \leqslant x} \frac{\Lambda(n)}{n}=\log x-\gamma+o(1) \quad(x \rightarrow \infty)
$$

to obtain the first claimed bound. The sum on $n$ equals

$$
\sum_{\substack{p \leqslant x \\ p \equiv 1(\bmod q)}} \frac{\log p}{p-1}-A(x)+B(x),
$$

where

$$
A(x)=\sum_{\substack{p \leqslant x, p^{a}>x \\ p \equiv 1(\bmod q)}} \frac{\log p}{p^{a}}, \quad B(x)=\sum_{\substack{p^{a} \leqslant x \\ p^{a} \equiv 1(\bmod q) \\ p \neq 1(\bmod q)}} \frac{\log p}{p^{a}}
$$

Clearly, $\lim _{x \rightarrow \infty} B(x)=S(q)$. The last estimate we need is $\lim _{x \rightarrow \infty} A(x)=0$, which is proved as follows:

$$
A(x) \leqslant \sum_{a=2}^{\infty} \sum_{n>x^{1 / a}} \frac{\log n}{n^{a}} \ll \sum_{a=2}^{\infty} \frac{\log x}{a^{2} x^{1-1 / a}} \ll \frac{\log x}{\sqrt{x}}
$$

Remark 1. Alternatively one can prove Proposition 3 by making the limit formula (1.16) explicit for $K(q)$ using Lemma 1 .

Remark 2. Proposition 3 can be used to approximate, nonrigorously, the value of $\gamma_{q}$. For example, when $q=964477901$, the right side in Proposition 3 stays very close to -0.18 for $10^{6} \leqslant x / q \leqslant 10^{7}$; see Theorem 4 .

Proposition 6. If $y \geqslant 10 q$ and $q \geqslant 11$, then

$$
\sum_{\substack{p \leqslant y \\ p \equiv 1(\bmod q)}} \frac{\log p}{p-1} \leqslant \frac{2 \log y+2(\log q) \log \log (y / q)}{q-1} .
$$

Proof. By the Montgomery-Vaughan sharpening of the Brun-Titchmarsh inequality [35], we have

$$
\pi(y ; q, 1) \leqslant \frac{2 y}{(q-1) \log (y / q)}
$$


and hence, by partial summation,

$$
\begin{aligned}
& \sum_{\substack{p \leqslant y \\
p \equiv 1(\bmod q)}} \frac{\log p}{p-1}=\frac{\pi(y ; q, 1) \log y}{y-1}+\int_{2 q}^{y} \frac{\pi(t ; q, 1)}{(t-1)^{2}}\left(\log t-\frac{t-1}{t}\right) d t \\
& \leqslant \frac{2}{q-1}\left(\frac{y \log y}{(y-1) \log (y / q)}+\int_{2 q}^{y} \frac{t}{(t-1)^{2}}+\frac{t \log q-(t-1)}{(t-1)^{2} \log (t / q)} d t\right) \\
& \leqslant \frac{2}{q-1}\left(\frac{y}{y-1}\left(1+\frac{\log q}{\log 10}\right)+\int_{2 q}^{y} \frac{1}{t}+\frac{2}{(t-1)^{2}}+\frac{\log q}{t \log (t / q)} d t\right) \\
& \leqslant \frac{2}{q-1}\left(1.01+0.44 \log q+\log \left(\frac{y}{2 q}\right)+\frac{2}{2 q-1}\right. \\
& \leqslant \frac{2 \log y+2(\log q) \log \log (y / q)}{q-1} .
\end{aligned}
$$

Proposition 7. Uniformly for $z \geqslant 2, \delta>0$ and $0<\varepsilon \leqslant 1$, the number of primes $q \leqslant z$ for which

is $O(\varepsilon \pi(z) / \delta)$.

$$
\sum_{\substack{p \leqslant q^{1+\varepsilon} \\ p \equiv 1(\bmod q)}} \frac{\log p}{p-1} \geqslant \delta \frac{\log q}{q}
$$

Proof. By sieve methods (e.g. [18, Theorem 5.7]), for an even $k \geqslant 2$, the number of prime $q \leqslant z$ with $k q+1$ prime is $O\left(\frac{k}{\phi(k)} \frac{z}{\log ^{2} z}\right)$ uniformly in $k$. Thus, the number of primes $q$ in question is

$$
\begin{aligned}
& \leqslant \sum_{q \leqslant z} \frac{q}{\delta \log q} \sum_{\substack{p \leqslant q^{1+\varepsilon} \\
p \equiv 1(\bmod q)}} \frac{\log p}{p-1} \leqslant \frac{1}{\delta} \sum_{\substack{k \leqslant z^{\varepsilon} \\
2 \mid k}} \sum_{\substack{k^{1 / \varepsilon} \leqslant q \leqslant z \\
k q+1 \text { prime }}} \frac{\log (k q+1)}{k \log q} \\
& \ll \frac{z}{\delta \log ^{2} z} \sum_{k \leqslant z^{\varepsilon}} \frac{1}{\phi(k)} \ll \frac{\varepsilon}{\delta} \frac{z}{\log z},
\end{aligned}
$$

where we used the well-known estimate $\sum_{n \leqslant x} \phi(n)^{-1}=O(\log x)$.

Lemma 3. Let $q \geqslant 10000$ be prime and let $\chi$ be the quadratic character modulo $q$. If $L\left(\beta_{0}, \chi\right)=0$, then

$$
\beta_{0} \geqslant 1-\frac{3.125 \min \left(2 \pi, \frac{1}{2} \log q\right)}{\sqrt{q} \log ^{2} q} .
$$

Proof. By Dirichlet's class number formula [5, §6, (15) and (16)],

$$
L(1, \chi)= \begin{cases}\frac{\pi h(-q)}{\sqrt{q}} & q \equiv 3(\bmod 4), \\ \frac{h(q) \log u}{\sqrt{q}} & q \equiv 1(\bmod 4),\end{cases}
$$

where $h(d)$ is the class number of $\mathbb{Q}(\sqrt{d})$, and $u$ is the smallest unit in $\mathbb{Q}(\sqrt{d})$ satisfying $u>1$. Since $u>\sqrt{q}$ and $h(-s) \geqslant 2$ for $s>163$, we obtain for $q>163$ that $L(1, \chi) \geqslant \min \left(2 \pi, \frac{1}{2} \log q\right) q^{-1 / 2}$. Assume $\beta_{0} \geqslant 1-0.2 q^{-1 / 2}$, otherwise there 
is nothing to prove. Let $V(t)=\sum_{n \leqslant t} \chi(n)$. By the Pólya-Vinogradov inequality ([5. $\S 23,(2)]$ or [36, $\S 9.4])$, for $t>u>0$,

$$
\begin{aligned}
|V(t)-V(u)| & <\frac{2}{\sqrt{q}} \sum_{a=1}^{(q-1) / 2} \frac{1}{\sin (\pi a / q)} \leqslant \frac{2}{\sqrt{q}} \int_{1 / 2}^{q / 2} \frac{d t}{\sin (\pi t / q)} \\
& =\frac{2 \sqrt{q}}{\pi} \log \cot \left(\frac{\pi}{4 q}\right)<\frac{2}{\pi} \sqrt{q} \log (4 q / \pi) .
\end{aligned}
$$

Hence, for $\frac{1}{2} \leqslant \sigma \leqslant 1$ and $y \geqslant 100$,

$$
\begin{aligned}
\left|L^{\prime}(\sigma, \chi)\right| & \leqslant y^{1-\sigma} \sum_{n \leqslant y} \frac{\log n}{n}+\int_{y}^{\infty}|V(t)-V(y)| \frac{\sigma \log t-1}{t^{1+\sigma}} d t \\
& \leqslant y^{1-\sigma}\left(\frac{\log ^{2} y}{2}+\frac{2}{\pi} \sqrt{q} \log \left(\frac{4 q}{\pi}\right) \frac{\log y}{y}\right) .
\end{aligned}
$$

Taking $y=q^{0.67}$ gives

$$
\left|L^{\prime}(\sigma, \chi)\right| \leqslant q^{0.67(1-\sigma)}\left(0.316 \log ^{2} q\right) \leqslant 0.32 \log ^{2} q .
$$

The mean value theorem implies $\left(1-\beta_{0}\right)\left(0.32 \log ^{2} q\right) \geqslant L(1, \chi)$ and the lemma follows.

3.2. Numerical calculation of $\gamma_{q}$. The identity (2.6) is useful for numerically calculating $\gamma_{q}$ for small $q$. For example (cf. 38]),

$$
\gamma_{3}=\gamma+\frac{L^{\prime}\left(1, \chi_{3}\right)}{L\left(1, \chi_{3}\right)}=0.945497280871680703239749994158189073 \ldots
$$

where $\chi_{3}$ stands for the only nonprincipal character modulo 3. For larger $q$ we use the following formulas. First,

$$
L(1, \chi)=-\frac{1}{q} \sum_{r=1}^{q-1} \chi(r) \psi\left(\frac{r}{q}\right), \quad \psi(z)=\frac{\Gamma^{\prime}(z)}{\Gamma(z)} .
$$

We also use

$$
-L^{\prime}(1, \chi)=\sum_{n=1}^{\infty} \frac{\chi(n) \log n}{n}=(\log q) L(1, \chi)+\frac{1}{q} \sum_{r=1}^{q-1} \chi(r) T\left(\frac{r}{q}\right),
$$

where

$$
T(y)=\sum_{m=0}^{\infty}\left(\frac{\log (m+y)}{m+y}-\frac{\log (m+1)}{m+1}\right) .
$$

Here, the term $(m+1)^{-1} \log (m+1)$ is a convergence factor, included so that the terms in the sum on $m$ are $O\left(m^{-2} \log m\right)$. The advantage of using (3.1) and (3.2) is that for each $q$, there are only $q-1$ values of $\psi$ and $q-1$ sums $T(r / q)$ to compute. With these values in hand, there are, however, still $\gg q^{2}$ operations (additions, subtractions, multiplications, divisions) needed using a naive algorithm to compute all of the numbers $L(1, \chi)$ and $L^{\prime}(1, \chi)$. A significant speed-up is achieved by observing that the vector of sums on $r$ on the right sides of (3.1) and (3.2) are discrete Fourier transform coefficients. Specifically, let $g$ be a primitive root of $q, \chi_{1}$ the character with $\chi_{1}(g)=e^{2 \pi i /(q-1)}$ and for $1 \leqslant k \leqslant q-1$, let $r_{k}$ be the integer in $[1, q-1]$ satisfying $g^{k} \equiv r_{k}(\bmod q)$. The characters modulo $q$ are $\chi_{0}, \chi_{1}, \chi_{1}^{2}, \ldots, \chi_{1}^{q-2}$ and for $\chi=\chi_{1}^{j}$, the sum in (3.1) is $\sum_{k=1}^{q-1} e^{2 \pi i j k /(q-1)} \psi\left(r_{k} / q\right)$ 


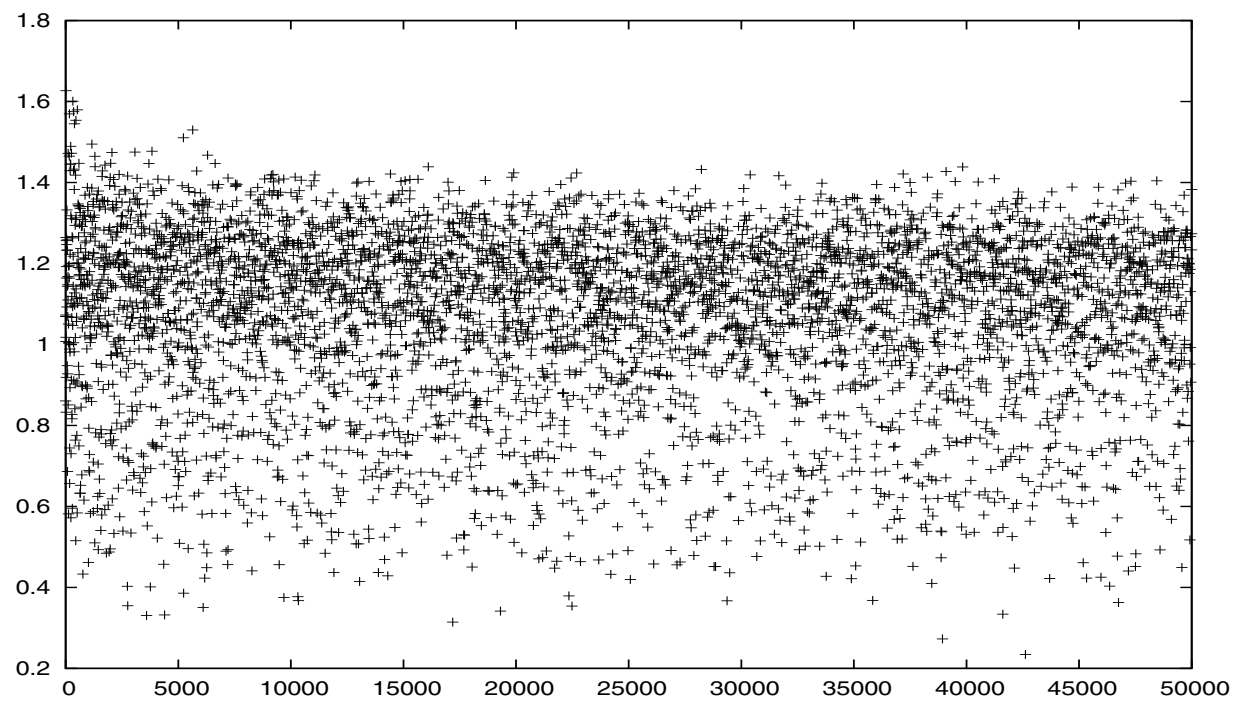

Figure 1. $\gamma_{q} / \log q$ for $q \leqslant 50000$

and the sum on $r$ in (3.2) is $\sum_{k=1}^{q-1} e^{2 \pi i j k /(q-1)} T\left(r_{k} / q\right)$. Fast Fourier Transform (FFT) algorithms may be used to recover $L(1, \chi)$ and $L^{\prime}(1, \chi)$ from the vectors $\left(\psi\left(r_{1} / q\right), \ldots, \psi\left(r_{q-1} / q\right)\right)$ and $\left(T\left(r_{1} / q\right), \ldots, T\left(r_{q-1} / q\right)\right)$, respectively, with $O(q \log q)$ operations.

A program to compute the numbers $L(1, \chi)$ and $L^{\prime}(1, \chi)$ was written in the $\mathrm{C}$ language, making use of the FFT library fftw [11. Running on a Dell Inspiron 530 desktop computer with Ubuntu Linux, 2GB RAM and a $2.0 \mathrm{GHz}$ processor, the program computed $\gamma_{q}$ for all prime $q \leqslant 30000$ in 2 minutes. All computations were performed using high precision arithmetic (80-bit "long double precision" floating point numbers). In order to handle very large $q$ (larger than about $5 \times 10^{7}$ ) a machine with more memory was required. A suitably modified version of the program was run on a large cluster computer, with 256GB RAM, 48 core AMD Opteron 6176 SE processors (4 sockets, 12 cores/socket), operating system Ubuntu Linux 10.04.3 LTS x86_64. The computation of $\gamma_{q}$ for $q=964477901$ took 64 minutes of CPU time on this system. This gave Theorem 4 .

Lemma 4. For $q \leqslant 30000$, we have $0.315 \log q \leqslant \gamma_{q} \leqslant 1.627 \log q$.

The largest value of $\gamma_{q} / \log q$ among $q \leqslant 30000$ is $\gamma_{19} / \log 19=1.626 \ldots$ and the smallest is $\gamma_{17183} / \log 17183=0.315 \ldots$ Lemma 4 suffices for the application to Theorem 1 .

In the next subsection, we will discuss more about the likely distribution of the Euler-Kronecker constants. Figure 1 displays a scatter plot of $\gamma_{q} / \log q$ for the primes $q \leqslant 50000$.

\subsection{Conditional bounds for $\gamma_{q}$.}

Lemma 5. (i) For all $C>0$ and for all except $O\left(\pi(u) /(\log u)^{C}\right)$ primes $q \leqslant u$,

$$
\gamma_{q}=2 \log q-q \sum_{\substack{p \leqslant q^{2} \\ p \equiv 1(\bmod q)}} \frac{\log p}{p-1}+O_{C}(\log \log q) .
$$


(ii) Assuming ERH, the above inequality holds for all prime $q$ (the implied constant in the $\mathrm{O}_{C}(\log \log q)$ term being absolute in this case).

(iii) Assume Conjecture EH, fix $C>0$ and $\varepsilon>0$. For all but $O\left(\pi(u) /(\log u)^{C}\right)$ primes $q \leqslant u$,

$$
\gamma_{q}=(1+\varepsilon) \log q-q \sum_{\substack{p \leqslant q^{1+\varepsilon} \\ p \equiv 1(\bmod q)}} \frac{\log p}{p-1}+O_{C}(\log \log q) .
$$

Proof. Part (i) follows by a straightforward combination of Proposition 3 and the Bombieri-Vinogradov theorem [5, §28] (cf. Proposition 2 of [15]). The latter states that for all $A>0$ there is a $B$ such that

$$
\sum_{q \leqslant \sqrt{x} / \log ^{B} x}|E(x ; q)| \ll \frac{x}{(\log x)^{A}} .
$$

For any $x \geqslant z>q$, partial summation implies

$$
\sum_{\substack{y \leqslant p \leqslant x \\ p \equiv 1(\bmod q)}} \frac{\log p}{p-1}=\frac{\log \left(\frac{x-1}{y-1}\right)}{q-1}+G_{q}(x, z),
$$

where

$$
G_{q}(x, z)=\left[\frac{E(x ; t) \log t}{t-1}\right]_{y}^{x}+\int_{y}^{x}\left(\frac{\log t}{(t-1)^{2}}-\frac{1}{t^{2}-t}\right) E(t ; q) d t .
$$

Let $B$ be the constant corresponding to $A=C+3$, let $z$ be large and put $y=z^{2}(\log z)^{2 B+1}$. For any $t \geqslant y, 2 z \leqslant \sqrt{t}(\log t)^{-B}$, and so

$$
S(t ; z):=\sum_{z<q \leqslant 2 z}|E(t ; q)| \ll \frac{t}{(\log t)^{C+3}} .
$$

We obtain

$$
\sum_{z<q \leqslant 2 z} \sup _{x>y}\left|G_{q}(y, x)\right| \ll \sup _{t \geqslant y} \frac{S(t ; z) \log t}{t}+\int_{y}^{\infty} \frac{S(t ; x) \log t}{t^{2}} d t \ll \frac{1}{(\log z)^{C+1}} .
$$

Thus, the summand on the left is $\geqslant 1 /(2 z)$ for $O\left(z(\log z)^{-C-1}\right)$ primes $q \in(z, 2 z]$. Summing over dyadic intervals, we find that

$$
\sup _{x>y}\left|G_{q}(y, x)\right| \geqslant \frac{1}{q}
$$

for $O\left(\pi(u) / \log ^{C} u\right)$ primes $q \leqslant u$. For the other (nonexceptional) $q$, from Proposition 3 and Theorem 3 we obtain

$$
\gamma_{q}=2 \log (y-1)+O(1)-(q-1) \sum_{\substack{p \leqslant y \\ p \equiv 1(\bmod q)}} \frac{\log p}{p-1},
$$

where $y \asymp q^{2}(\log q)^{2 B+1}$. Finally, the Brun-Titchmarsh inequality and partial summation gives

$$
\sum_{\substack{q^{2}<p \leqslant y \\ p \equiv 1(\bmod q)}} \frac{\log p}{p-1} \ll \frac{\log \left(y / q^{2}\right)}{q} \ll_{C} \frac{\log \log q}{q} .
$$


This proves (i). To obtain (ii), insert into (3.3) the bound $E(t ; q) \ll \sqrt{t} \log q$ valid under ERH (apply partial summation to [5, $\S 20,(14)]$ ), take $y=q^{2}(\log q)^{C+10}$ and argue as in part (i). To prove (iii), substitute Conjecture EH for the BombieriVinogradov Theorem and take $y=z^{1+\varepsilon}$ in the above argument.

Part (ii) of Lemma 5 may also be deduced from a general bound for $\gamma_{K}$ due to Ihara [22, Proposition 2].

Lemma 6. For any $M>0$, there is an admissble set $\left\{a_{1}, \ldots, a_{k}\right\}$ with $\sum_{i} 1 / a_{i}>M$.

Proof. Let $p_{1}=3$ and, recursively for each $k \geqslant 2$, let $p_{k}$ be the smallest prime for which $p_{k} \not \equiv 1\left(\bmod p_{j}\right)$ for all $j<k$. Thus $p_{2}=5, p_{3}=17, p_{4}=23$, etc. Erdös in [10], answering a question of $\mathrm{S}$. Golomb, proved that $\sum_{k=1}^{\infty} 1 / p_{k}$ diverges. For a given $M$, let $J$ be so large that if $\mathcal{B}=\left\{2\left(p_{j}+1\right): 1 \leqslant j \leqslant J\right\}$, then $\sum_{b \in \mathcal{B}} 1 / b>M$. We now deduce that $\mathcal{B}$ is admissible. Let $F(n)=n \prod_{b \in \mathcal{B}}(b n+1)$. Observe that by construction, if $r$ is prime and $r=p_{j}$ for some $j$, then none of the elements of $\mathcal{B}$ are congruent to $2(\bmod r)$. Hence, if $4 n \equiv-1(\bmod r)$, then $r \nmid F(n)$. If $r$ is a prime and $r \neq p_{j}$ for every $j$, then none of the elements of $\mathcal{B}$ are congruent to 1 $(\bmod r)$. Consequently, if $2 n \equiv-1(\bmod r)$, then $r \nmid F(n)$.

According to Granville [15], Lemma 6] was conjectured by Erdős in 1988. A proof is given in [15, Theorem 3]. We showed above that Lemma 6 is actually a simple corollary of Erdős' 1961 paper [10].

Proof of Theorem 5 and Proposition 4. Let $M \geqslant 0$ be arbitrary. Using Lemma 6. there is an admissible set $\left\{a_{1}, \ldots, a_{k}\right\}$ so that $\sum_{i} 1 / a_{i}>M+2$. By Lemma 5 (i), for all but $O\left(u / \log ^{k+2} u\right)$ primes $q \leqslant u$,

$$
\gamma_{q}=2 \log q+O_{M}(\log \log q)-q \sum_{\substack{p \leqslant q^{2} \\ p \equiv 1(\bmod q)}} \frac{\log p}{p-1} .
$$

Assuming Conjecture HL, there are $\gg u / \log ^{k+1} u$ primes $q \leqslant u$ for which $a_{i} q+1$ is prime for $1 \leqslant i \leqslant k$. For such primes $q>a_{k}+1$,

$$
q \sum_{\substack{p \leqslant q^{2} \\ p \equiv 1(\bmod q)}} \frac{\log p}{p-1} \geqslant \sum_{i=1}^{k} \frac{\log q}{a_{i}}>(M+2) \log q .
$$

Theorem 5 follows.

Proposition 4 follows by taking $M=0$ in the above argument and noting that we may take an admissible set with $k=2089$.

Proof of Theorem 6. Fix $\eta>0$. Assuming Conjecture EH and using Lemma 5 (iii), we see that for all but $O\left(\pi(u) / \log ^{C} u\right)$ primes $q \leqslant u$,

$$
\gamma_{q}=\left(1+\eta^{2}\right) \log q+O_{C}(\log \log q)-q \sum_{\substack{p \leqslant q^{1+\eta^{2}} \\ p \equiv 1(\bmod q)}} \frac{\log p}{p-1} .
$$

On the other hand, by Lemma 7 (with $\delta=\eta / 2$ and $\varepsilon=\eta^{2}$ ), for all but $O(\eta \pi(u)$ ) primes $p \leqslant u$, the above sum on $p$ is $\leqslant(\eta \log q) / q$. Hence, taking $C=1$, for all but $O(\eta \pi(u))$ primes $p \leqslant u,(1-\eta) \log q \leqslant \gamma_{q} \leqslant(1+\eta) \log q$ for large enough $q$. As $\eta$ is arbitrary, part (i) follows. 
To show part (ii) concerning limit points of $\gamma_{q} / \log q$, start with (3.4) and let $\varepsilon=\eta^{2}$. Let $\mathcal{A}=\left\{a_{1}, \ldots, a_{k}\right\}$ be an admissible set and let $m(\mathcal{A})=\sum_{i} 1 / a_{i}$. Assuming Conjecture HL, there are $\gg u / \log ^{k+1} u$ primes $q \leqslant u$ such that $a_{i} q+1$ is prime for $1 \leqslant i \leqslant k$. By sieve methods [18, Theorem 5.7], the number of primes $q \leqslant u$ for which $a_{i} q+1$ is prime $(1 \leqslant i \leqslant k)$ and $b q+1$ is also prime is $O\left(\frac{b}{\phi(b)} u / \log ^{k+2} u\right)$, where the implied constant depends on $\mathcal{A}$. Summing over even $b \leqslant q^{\varepsilon}, b \in \mathcal{A}$, we find that there are $O\left(\varepsilon u / \log ^{k+1} u\right)$ primes $q \leqslant u$ with $b q+1$ prime for some $b \leqslant q^{\varepsilon}, b \notin \mathcal{A}$. If $\varepsilon$ is small enough, depending on $\mathcal{A}$, then there are $\gg u / \log ^{k+1}$ primes $q \leqslant u$ for which $q a_{i}+1$ is prime $(1 \leqslant i \leqslant k)$ and $q b+1$ is composite for all $b \leqslant q^{\varepsilon}$ such that $b \notin \mathcal{A}$. For such $q$, (3.4) with $C=k+2$ implies that

$$
\gamma_{q}=(1+\varepsilon-m(\mathcal{A})) \log q+O_{k}(\log \log q) .
$$

As $\varepsilon$ is arbitrary, we see that $1-m(\mathcal{A})$ is a limit point of $\left\{\gamma_{q} / \log q: q\right.$ prime $\}$. Finally, it follows immediately from Lemma 6 that $\{m(\mathcal{A}): \mathcal{A}$ admissible $\}$ is dense in $[0, \infty)$. Indeed, given any $x>0$ and $\delta>0$, there is an admissible set of integers $>1 / \delta$ with $m(\mathcal{A})>x$. As any subset of an admissible set is also admissible, there is a subset $\mathcal{A}^{\prime}$ of $\mathcal{A}$ with $\left|m\left(\mathcal{A}^{\prime}\right)-x\right|<\delta$.

\section{UPPER BOUNDS FOR $S(q)$}

We will give explicit upper bounds in Theorem 3 for $S(q)$, making use of explicit estimates for prime numbers from [45]. Note that $f_{p} \geqslant 2$ implies that $q \mid\left(p^{f_{p}}-1\right) /(p-1)$, that is,

$$
\frac{p^{f_{p}}-1}{p-1}=q n_{p}, \quad n_{p} \geqslant 1 .
$$

Lemma 7. For $x \geqslant 2$,

$$
\log x-0.605 \leqslant \sum_{p \leqslant x} \frac{\log p}{p-1} \leqslant \begin{cases}\log x-0.142 & (x \geqslant 9) \\ \log x-\frac{1}{2} & (x \geqslant 467.4)\end{cases}
$$

Also,

$$
\sum_{p \geqslant x} \frac{\log p}{p^{3}-1} \leqslant \frac{0.6}{x^{2}} \quad(x>2) .
$$

Proof. For the first estimate, we note that

$$
\sum_{p \leqslant x} \frac{\log p}{p-1}=\sum_{p \leqslant x} \frac{\log p}{p}+\sum_{p \leqslant x} \frac{\log p}{p(p-1)} .
$$

The latter sum can be easily bounded by 0.756 . The first estimate then is derived by invoking [45. Theorems 6,21$]$ to deal with $x \geqslant 1000$ and direct numerical calculation for smaller $x$. For $x \geqslant 7481$ one has $0.98 x \leqslant \sum_{p \leqslant x} \log p \leqslant 1.01624 x$, as was shown by Rosser and Schoenfeld [45, Theorems 9 and 10]. From this, one easily infers that for $x \geqslant 7481$,

$$
\sum_{p>x} \frac{\log p}{p^{3}-1} \leqslant \frac{x}{x^{3}-1}\left(-0.98+1.01624\left(\frac{3}{2}\right) \frac{x^{3}}{\left(x^{3}-1\right)}\right) .
$$


For $k=2$, the right side is $\leqslant 1.0525 x^{-1}$ and for $k=2$, the right side is $\leqslant 0.545 x^{-2}$. For $x<7481$, we explicitly calculate the sum using

$$
\sum_{p>x} \frac{\log p}{p^{3}-1}=-\frac{\zeta^{\prime}(3)}{\zeta(3)}-\sum_{p \leqslant x} \frac{\log p}{p^{3}-1} .
$$

\subsection{A simple upper bound.}

Lemma 8. Let $q$ be a prime with $q \geqslant 5$. We have

$$
S(q) \leqslant \frac{\log q+1}{2 q} .
$$

Proof. First, if $f_{p}=2$, then $p=2 k q-1$ for a positive integer $k$. As $p \geqslant 13$, we have $p^{2}-1 \geqslant 6(p+1)^{2} / 7$. Thus,

$$
\begin{aligned}
\sum_{p \equiv-1(\bmod q)} \frac{\log p}{p^{2}-1} & \leqslant \frac{7}{6} \sum_{k=1}^{\infty} \frac{\log (2 k q)}{4 k^{2} q^{2}} \\
& =\frac{(7 / 6)\left(\zeta(2) \log (2 q)-\zeta^{\prime}(2)\right)}{4 q^{2}} \leqslant \frac{0.48 \log q+0.61}{q^{2}} .
\end{aligned}
$$

Next, suppose $p>q$ and $f_{p} \geqslant 3$. Combining the latter estimate and Lemma 7 we conclude that

$$
\sum_{p>q, f_{p} \geqslant 2} \frac{\log p}{p^{f_{p}}-1} \leqslant \frac{0.48 \log q+1.21}{q^{2}} .
$$

Now suppose $p<q$ (so that $f_{p} \geqslant 3$ ). If $q \geqslant 83$, by Lemma 7 , we have

$$
S^{\prime}(q)=\sum_{p<q} \frac{\log p}{p^{f_{p}}-1} \leqslant \frac{1}{q} \sum_{p<\sqrt{q}} \frac{\log p}{p-1}+\sum_{p>\sqrt{q}} \frac{\log p}{p^{3}-1} \leqslant \frac{0.5 \log q+0.458}{q} .
$$

By combining this estimate with (4.2) yields the claimed bound for $q \geqslant 83$. For $5 \leqslant q<83$, direct calculation shows that $S^{\prime}(q) \leqslant \frac{\log q-0.5}{2 q}$ and the claimed bound on $S(q)$ follows from (4.2).

Lemma 8 is strong enough in order to prove Theorem 1. However, with a refined analysis, we can obtain a sharper inequality when $q$ is large.

4.2. Refined upper bound. Note that in case $q$ is a Mersenne prime we have

$$
S(q) \geqslant \frac{\log 2}{2^{f_{2}}-1}=\frac{\log 2}{q} .
$$

Actually, the only $q$ we have been able to find for which $S(q)>(\log 2) / q$ are the Mersenne primes. It is thus conceivable that if $q$ is not a Mersenne prime, then always $S(q)<(\log 2) / q$. For a given $\epsilon>0$ it also appeared to us that the primes $q$ for which $S(q)>\epsilon / q$ have density zero. In what follows, we prove that this is the case. In general $S(q)$ is relatively large if $q$ almost equals a number of the form $p^{r}-1$ with $p$ small. For example, if $2 q=3^{r}-1$ for some $r$ (e.g. when $r=3,7,13,71$ ), then $S(q)>(\log 3) /(2 q)$. The above remarks show that the upper bound in the first part of Theorem 3, except for the constant, is likely optimal.

Proof of Theorem 3. We prove both (a) and (b) simultaneously. If $5 \leqslant q \leqslant 10^{30}$, Lemma 8 gives $S(q)<35.1 / q$ and (a) follows. Now suppose $q>10^{30}$. We first consider three ranges for $p$ : 
(i) $p>q$,

(ii) $p<q$ and $f_{p} \leqslant F=\left\lceil\frac{\log q}{3 \log \log q}\right\rceil$,

(iii) $f_{p} \geqslant F+1$ and $p>\log ^{4} q$.

Inequality (4.2) gives a good bound for the contribution of the primes in the range (i) to $S(q)$. Note that given $f \geqslant 3$, there are at most $f-1$ primes $p<q$ with $f_{p}=f$. By (4.1), $q \leqslant 2 p^{f-1}$, hence the contribution to $S(q)$ from a given $f$ is

$$
\leqslant \frac{(f-1) \log \left[(q / 2)^{1 /(f-1)}\right]}{(q / 2)^{f /(f-1)}-1} \leqslant 2.83 \frac{\log q}{q^{1+\frac{1}{f-1}}} .
$$

If $f \leqslant F$, then $q^{\frac{1}{f-1}} \geqslant \log ^{3} q$ and the contribution to $S(q)$ from such $f$ is

$$
\leqslant \frac{2.83(F-2)}{q \log ^{2} q} \leqslant \frac{2.83}{3 q(\log q) \log \log q} \text {. }
$$

For $p$ counted in the range (iii), $p^{f_{p}-1} \geqslant p^{F} \geqslant q^{4 / 3}$. By Lemma 7 the contribution to $S(q)$ from such $p$ is

$$
\leqslant \frac{1}{q^{4 / 3}} \sum_{\log ^{4} 4<p<q} \frac{\log p}{p-1} \leqslant \frac{\log q}{q^{4 / 3}} .
$$

By (4.2), (4.3) and (4.4), the contribution to $S(q)$ from $p$ in ranges (i)-(iii) is

$$
O\left(\frac{1}{q \log q}\right) \quad \text { and also } \leqslant \frac{1}{310 q} \text {. }
$$

The primes $p$ not considered in ranges (i)-(iii) satisfy $p \leqslant \log ^{4} q$ and $f_{p}>F$. We now take a brief interlude to prove (b). The contribution to $S(q)$ from those $p$ with $f_{p} \geqslant F^{\prime}=\left\lceil\frac{2 \log q}{\log 2}\right\rceil$ is $\leqslant 2 \sum_{p}(\log p) p^{-F^{\prime}}=O\left(q^{-2}\right)$. As $f_{p} \mid(q-1)$, we have dealt with all ranges unless $q-1$ has a divisor in $\left(F, F^{\prime}\right)$. But this is rare; specifically, by Theorems 1 and 6 of [16], the number of $q \in(x, 2 x]$ with such a divisor is $O\left(\pi(x)(\log \log \log x / \log \log x)^{-0.086}\right)$. By (4.5), (b) follows.

Next, we continue proving (a), by considering further ranges:

(iv) $p \leqslant e^{41}$,

(v) $e^{41}<p \leqslant \log ^{4} q$ and $n_{p} \geqslant \min \left(p, f_{p}\right)$,

(vi) $e^{41}<p \leqslant \log ^{4} q$ and $n_{p}<\min \left(p, f_{p}\right)$.

Trivially, by Lemma 7 , the contribution to $S(q)$ in case (iv) is

$$
\leqslant \frac{1}{q} \sum_{p \leqslant e^{41}} \frac{\log p}{p-1} \leqslant \frac{40.5}{q} .
$$

For ranges (v) and (vi), observe that $\log q \geqslant e^{41 / 4}$. Since $f_{p} \geqslant \frac{\log q}{\log p}$, the contribution to $S(q)$ in case $(\mathrm{v})$ is

$$
\begin{aligned}
& \leqslant \frac{1}{q \log q} \sum_{e^{41}<p \leqslant \log ^{4} q} \frac{\log ^{2} p}{p-1}+\sum_{p>e^{41}} \frac{\log p}{q p(p-1)} \\
& \leqslant \frac{4 \log \log q(4 \log \log q-40.895)}{q \log q}+\frac{10^{-10}}{q} \leqslant \frac{1}{416 q} .
\end{aligned}
$$

Here we used again Lemma 7 , together with the fact that the maximum of $x(x-b) e^{-x / 4}$ occurs at $x=\left(b+8+\sqrt{b^{2}+64}\right) / 2($ here $x=4 \log \log q)$. 
Now consider range (vi). We will show that $f_{p}$ is prime. Indeed, assume that $f_{p}$ is composite. Then

$$
\frac{p^{f_{p}}-1}{p-1}=\prod_{\substack{d \mid f_{p} \\ d>1}} \Phi_{d}(p),
$$

where $\Phi_{d}(X) \in \mathbb{Z}[X]$ is the $d$ th cyclotomic polynomial. There exists some divisor $d_{0}>1$ of $f_{p}$ such that $q \mid \Phi_{d_{0}}(p)$ (in fact, $d_{0}=f_{p}$, but this is not needed for the proof). Hence,

$$
n_{p} \geqslant \prod_{\substack{d \mid f_{p} \\ d \neq 1, d_{0}}} \Phi_{d}(p) .
$$

Since $f_{p}$ is not prime, the number $f_{p}$ has at least three divisors. Let $d_{1}>1$ be any divisor of $f_{p}$ different from $d_{0}$. Then

$$
n_{p} \geqslant \Phi_{d_{1}}(p)>(p-1)^{\phi\left(d_{1}\right)} \geqslant p-1,
$$

so $n_{p} \geqslant p$, a contradiction. Hence, $f=f_{p}$ is a prime factor of $q-1$. By Fermat's Little Theorem, $p^{f} \equiv p(\bmod f)$. Further, if $p \equiv 1(\bmod f)$, then $\left(p^{f}-1\right) /(p-1)$ is a multiple of $f$. Otherwise, $p-1$ is invertible modulo $f$, and since $p^{f}-1 \equiv p-1(\bmod f)$, we get that $\left(p^{f}-1\right) /(p-1)$ is congruent to 1 modulo $f$. Hence,

$$
q n_{p}=\frac{p^{f}-1}{p-1} \equiv 0,1(\bmod f),
$$

and since $q \equiv 1(\bmod f)$, we conclude that $n_{p} \equiv 0,1(\bmod f)$. But $n_{p}<f$, hence $n_{p}=1$ and

$$
\frac{p^{f}-1}{p-1}=q
$$

By writing the left-hand side as $\sum_{j=0}^{f-1} p^{j}$, we see that, in particular, $p \mid(q-1)$. Since $q-1$ has at most $\frac{\log q}{\log \log q}$ prime factors $>\log q$, the contribution to $S(q)$ from $p \in\left(\log q, \log ^{4} q\right]$ is

$$
\leqslant \frac{\log q}{q \log \log q} \cdot \frac{4 \log \log q}{\log q-1} \leqslant \frac{4.004}{q} .
$$

Let $\mathcal{P}$ be the set of primes satisfying (4.8) which are in the interval $\left(e^{41}, \log q\right]$. We cover the interval in dyadic intervals of the form $\mathcal{I}_{k}=\left[2^{k}, 2^{k+1}\right)$ with $2^{k} \leqslant \log q$, and we look at $\mathcal{P}_{k}=\mathcal{P} \cap \mathcal{I}_{k}$. We will show below that $\mathcal{P}_{k}$ has at most one element, and hence

$$
\frac{1}{q} \sum_{p \in \mathcal{P}} \frac{\log p}{p} \leqslant \frac{1}{q} \sum_{k \geqslant 59} \frac{k \log 2}{2^{k}-1} \leqslant \frac{1}{10^{15} q} .
$$

Combined with (4.5), (4.6), (4.7) and (4.9), this proves the theorem.

Now assume that $\mathcal{P}_{k}$ has at least two elements for some $k$, so that $k \geqslant 59$. Let $p_{1}<p_{2}$ be any two elements in $\mathcal{P}_{k}$ with

$$
q=\frac{p_{1}^{f_{1}}-1}{p_{1}-1}=\frac{p_{2}^{f_{2}}-1}{p_{2}-1} .
$$

Since the function $f \mapsto\left(p^{f}-1\right) /(p-1)$ is increasing for all fixed $p$, it follows that $f_{1}>f_{2}$. Now

$$
\left(p_{2}-1\right) p_{1}^{f_{1}}-\left(p_{1}-1\right) p_{2}^{f_{2}}=p_{2}-p_{1}
$$


Thus,

$$
\left|\frac{\left(p_{1}-1\right)}{\left(p_{2}-1\right)} p_{2}^{f_{2}} p_{1}^{-f_{1}}-1\right|=\frac{p_{2}-p_{1}}{\left(p_{2}-1\right) p_{1}^{f_{1}}}<\frac{1}{p_{1}^{f_{1}}} \leqslant \frac{1}{2^{k f_{1}}} .
$$

On the left, we use a lower bound for a linear form in three logarithms. Note that since $p_{2}>p_{1}$ this expression is not zero. Now all three rational numbers $\left(p_{1}-1\right) /\left(p_{2}-1\right), p_{1}$ and $p_{2}$ have height $<2^{k+1}$. Thus, Matveev's bound from 33 . (see also Theorem 9.4 in [4]) tells us at once that

$$
\begin{aligned}
\log \left|\frac{\left(p_{1}-1\right)}{\left(p_{2}-1\right)} p_{2}^{f_{2}} p_{1}^{-f_{1}}-1\right| & >-1.4 \times 30^{6} \times 3^{4.5}\left(1+\log \left(4 f_{1}\right)\right)\left(\log \left(2^{k+1}\right)\right)^{3} \\
& >-4.77 \times 10^{10}(k+1)^{3}\left(1+\log \left(4 f_{1}\right)\right) .
\end{aligned}
$$

Thus, comparing bounds (4.11) and (4.12), we get that

$$
k f_{1} \log 2<4.77 \times 10^{10}(k+1)^{3}\left(1+\log \left(4 f_{1}\right)\right) .
$$

Since $k \geqslant 59$,

$$
f_{1}<\frac{4.77 \times 10^{10}}{\log 2}\left(\frac{k+1}{k}\right)(k+1)^{2}\left(1+\log \left(4 f_{1}\right)\right)<7 \times 10^{10}(k+1)^{2} \log \left(4 f_{1}\right) .
$$

Here, we used the fact that $\log \left(4 f_{1}\right) \geqslant \log (4 F)>37$, so $1+\log \left(4 f_{1}\right)<\frac{38}{37} \log \left(4 f_{1}\right)$. This gives

$$
4 f_{1}<2.876 \times 10^{11}(k+1)^{2} \log \left(4 f_{1}\right) .
$$

For $A>10^{12}$, the inequality $x<A \log x$ implies that $x<\frac{9}{8} A \log A$ and hence

$$
f_{1}<8.1 \times 10^{10}(k+1)^{2}(26.4+2 \log (k+1)) .
$$

Since $f_{1} \log p_{1}>\log q, \log p_{1}<(k+1) \log 2$ and $2^{k} \leqslant \log q$, we have

$$
2^{k} \leqslant \log q<(\log 2) \times 10^{11}(k+1)^{3}(26.6+2 \log (k+1)) .
$$

This implies $k \leqslant 58$, a contradiction.

\section{Proof of Theorems 2 and 1}

Let

$$
E_{q}(t)=\Psi(t ; q, 1)-\frac{t}{q-1}, \text { where } \Psi(t ; q, 1)=\sum_{\substack{n \leqslant x \\ n \equiv 1(\bmod q)}} \Lambda(n) .
$$

Let $R=9.645908801$. We say that $\beta_{0}$ is an exceptional zero for a prime $q$ if $\beta_{0} \geqslant 1-1 /(R \log q)$ and $L\left(\beta_{0}, \chi\right)=0$, where $\chi$ is the quadratic character modulo $q$. Let $B(q)=1$ if $\beta_{0}$ exists, and $B(q)=0$ otherwise.

Lemma 9. Suppose $q \geqslant 10000$ is prime. Then, for $x \geqslant e^{R \log ^{2} q}$,

$$
\left|E_{q}(x)\right| \leqslant \frac{1.012 x^{\beta_{0}}}{q} B(q)+\frac{8}{9} x \sqrt{\frac{\log x}{R}} \exp \left\{-\sqrt{\frac{\log x}{R}}\right\} .
$$

The proof of Lemma 9 comes from estimates in McCurley [34, and will be given later in Section 6 . 
Proof of Theorem 2. Propositions 2 and 3 imply that

$$
(q-1) \frac{e_{1}(q)}{e_{0}(q)}=1-\gamma-\frac{2 \log q}{q^{2}-1}-S(q)+\lim _{x \rightarrow \infty}\left[\frac{\log x}{q-1}-\sum_{\substack{n \leqslant x \\ n \equiv 1(\bmod n)}} \frac{\Lambda(n)}{n}\right]
$$

By partial summation, for any $y>2 q$ we have

$$
\lim _{x \rightarrow \infty}\left(\sum_{\substack{y<n \leqslant x \\ n \equiv 1(\bmod q)}} \frac{\Lambda(n)}{n}-\frac{\log (x / y)}{q-1}\right)=-\frac{E_{q}(y)}{y}+\int_{y}^{\infty} \frac{E_{q}(t)}{t^{2}} d t .
$$

By Lemma 9 ,

$$
\begin{aligned}
\left|\int_{y}^{\infty} \frac{E_{q}(t)}{t^{2}} d t-\frac{E_{q}(y)}{y}\right| \leqslant B(q) \frac{1.012\left(2-\beta_{0}\right) y^{\beta_{0}-1}}{\left(1-\beta_{0}\right) q} \\
+\frac{8}{9}\left(\frac{2 R W^{2}+(4 R+1) W+4 R}{e^{W}}\right),
\end{aligned}
$$

where $W=\sqrt{\frac{\log y}{R}}$.

Taking $y=\exp \left(4 R \log ^{2} q\right)$ (so that $W=2 \log q$ ), we obtain

$$
\left|(q-1) \frac{e_{1}(q)}{e_{0}(q)}-(1-\gamma)\right| \ll \frac{\log y}{q}+\frac{B(q)}{1-\beta_{0}}+\sum_{\substack{n \leqslant y \\ n \equiv 1(\bmod q)}} \frac{\Lambda(n)}{n} .
$$

By Proposition [6] and Theorem 3, the above sum on $n$ is

$$
\leqslant S(q)+\frac{2 \log y+2(\log q) \log \log (y / q)}{q-1} \ll \frac{\log ^{2} q}{q} .
$$

The first three parts of Theorem 2 now follow: for the first part, use Lemma 3 , for the second part use Siegel's theorem [5, §21] which states that for every $\varepsilon>0$, $\beta_{0} \geqslant 1-C(\varepsilon) q^{-\varepsilon}$ for an (ineffective) constant $C(\varepsilon)$; for the third part, we assume $\beta_{0}$ doesn't exist.

Finally, on ERH we have $E_{q}(t) \ll t^{1 / 2} \log ^{2} t$, uniformly in $q \leqslant t[5$, §20, (14)]. Hence, if $y \geqslant q$, then

$$
\left|\int_{y}^{\infty} \frac{E_{q}(t)}{t^{2}} d t-\frac{E_{q}(y)}{y}\right| \ll \frac{\log ^{2} y}{y^{1 / 2}} .
$$

Taking $y=q^{3}$ in the above argument yields $\gamma_{q}=O((\log q)(\log \log q))$ and hence the final estimate in Theorem 2 ,

Remarks. The estimate $\gamma_{q}=O((\log q) \log \log q)$, valid under ERH, was proved independently by Badzyan [2]. Note that a third way to establish it is by using [22, Proposition 2]. Unconditionally, Ihara et al. 24] have shown that $\gamma_{q} \ll_{\varepsilon} q^{\epsilon}$ (implicit in the third estimate in Theorem 2). In a more recent paper [41, Kumar Murty proved that $\left|\gamma_{q}\right|$ is $O(\log q)$ on average:

$$
\sum_{Q / 2<q \leqslant Q}\left|\gamma_{q}\right| \ll(\pi(Q)-\pi(Q / 2)) \log Q .
$$


Proof of Theorem 1. By (5.1)-(5.3) (ignoring the summands in (5.1) with $n \leqslant y$ ), together with the exceptional zero estimate in Lemma 3, we have for $q \geqslant 10000$ the estimate

$$
\begin{aligned}
(q-1) \frac{e_{1}(q)}{e_{0}(q)} \leqslant 1-\gamma+\frac{\log y}{q-1}+1.015 & \frac{y^{-D /\left(q^{1 / 2} \log ^{2} q\right)} \log ^{2} q}{D q^{1 / 2}} \\
& +\frac{8}{9}\left(\frac{2 R W^{2}+(4 R+1) W+4 R}{e^{W}}\right),
\end{aligned}
$$

where $D=3.125 \max \left(2 \pi, \frac{1}{2} \log q\right)$. When $q \geqslant 30000$, we take $y=e^{1.44 R \log ^{2} q}$, so that $W=1.2 \log q$ and $D \geqslant 16.1$. A short calculation reveals that $e_{1}(q) / e_{0}(q)<\frac{1}{2}$.

For $q<30000$ we use the results of explicit calculation of $\gamma_{q}$ (e.g., Table 1 and Lemma 41).

\section{Proof of Lemma 9}

In [34, McCurley gives estimates for $E_{q}(x)$ under the assumption that the exceptional zero $\beta_{0}$ doesn't exist. It is simple to modify the arguments to handle the case when $\beta_{0}$ does exist. Define

$$
L=\log q, \quad X=\sqrt{\frac{\log x}{R}}, \quad x=e^{\lambda R L^{2}}, \quad \lambda=(1+\alpha)^{2}, \quad H=q^{\alpha} .
$$

In particular,

$$
X=(1+\alpha) L=\log (q H) .
$$

Also, since $q \geqslant 10000$, we have $x \geqslant 10^{355}$. We take $\eta=\frac{1}{2}$ in [34, Theorem 2.1], which gives

$$
\left|N(T, \chi)-\frac{T}{\pi} \log \left(\frac{q T}{2 \pi e}\right)\right| \leqslant C_{1} \log (q T)+C_{2},
$$

where $C_{1}=0.9185, c_{2}=5.512$ and $N(T, \chi)$ is the number of zeros of $L(s, \chi)$ with imaginary part in $[-T, T]$ and real part in $(0,1)$. Lemma 3.5 of $[34$ concerns bounds for $\sum_{\chi \neq \chi_{0}}|b(\chi)|$ (where $b(\chi)$ is the constant term in the Laurent expansion of $\frac{L^{\prime}}{L}(s, \chi)$ about $\left.s=0\right)$ and it is assumed that $\beta_{0}$ doesn't exist. However, by [34, (3.16)], the existence of $\beta_{0}$ contributes an extra amount $\leqslant \frac{1}{14} q^{1 / 2} \log ^{2} q$ to the sum. The estimate in this lemma is thus increased by an amount $\leqslant 0.06$ if $\beta_{0}$ exists.

We apply [34, Theorem 3.6] with $m=2$ and $\delta=2 / H \leqslant 0.0002$. In the notation of this theorem,

$$
A_{2}(\delta)=\delta^{-2}\left(1+2(1+\delta)^{3}+(1+2 \delta)^{3}\right) \leqslant 4.003 \delta^{-2} .
$$

Denote by $\rho=\beta+i \gamma$ a generic zero of a nonprincipal $L$-function with $0<\beta<1$. Then we have

$$
\begin{aligned}
& \frac{q-1}{x}\left|E_{q}(x)\right|<(1+\delta) \sum_{\chi \neq \chi_{0}} \sum_{\rho:|\gamma| \leqslant H} \frac{x^{\beta-1}}{|\rho|} \\
& \quad+\frac{4.003}{\delta^{2}} \sum_{\chi \neq \chi_{0}} \sum_{\rho:|\gamma|>H} \frac{x^{\beta-1}}{|\rho(\rho+1)(\rho+2)|}+\delta+\varepsilon_{1},
\end{aligned}
$$


TABle 1. Approximate values of $S(q), \gamma_{q}$ and $e_{1}(q) / e_{0}(q)$.

\begin{tabular}{|r|r|r||r|r||r|}
\hline$q$ & $S(q)$ & $q S(q)$ & $\gamma_{q}$ & $\gamma_{q} / \log q$ & $(q-1) \frac{e_{1}(q)}{e_{0}(q)}$ \\
\hline \hline 3 & 0.351646 & 1.054940 & 0.945497 & 0.860628 & 1.247179 \\
5 & 0.077777 & 0.388887 & 1.720624 & 1.069083 & 0.897187 \\
7 & 0.122829 & 0.859805 & 2.087594 & 1.072811 & 0.866519 \\
11 & 0.009100 & 0.100103 & 2.415425 & 1.007310 & 0.657441 \\
13 & 0.046201 & 0.600623 & 2.610757 & 1.017859 & 0.673826 \\
17 & 0.004437 & 0.075432 & 3.581976 & 1.264280 & 0.642487 \\
19 & 0.011009 & 0.209173 & 4.790409 & 1.626934 & 0.692657 \\
23 & 0.000829 & 0.019080 & 2.611289 & 0.832815 & 0.536910 \\
29 & 0.000347 & 0.010088 & 3.093731 & 0.918758 & 0.529900 \\
31 & 0.036585 & 1.134139 & 4.314442 & 1.256394 & 0.599845 \\
37 & 0.000929 & 0.034387 & 4.304938 & 1.192200 & 0.540802 \\
41 & 0.000449 & 0.018445 & 3.971521 & 1.069461 & 0.520422 \\
43 & 0.000218 & 0.009397 & 4.378627 & 1.164157 & 0.525317 \\
47 & 0.000129 & 0.006083 & 4.799394 & 1.246548 & 0.525580 \\
53 & 0.000214 & 0.011346 & 4.337736 & 1.092548 & 0.505056 \\
59 & 0.000065 & 0.003863 & 5.433516 & 1.332548 & 0.515399 \\
61 & 0.001438 & 0.087727 & 5.071085 & 1.233578 & 0.507672 \\
67 & 0.000268 & 0.018017 & 5.292139 & 1.258626 & 0.502328 \\
\hline 71 & 0.000612 & 0.043471 & 5.255258 & 1.232853 & 0.497650 \\
73 & 0.001374 & 0.100374 & 4.066949 & 0.947905 & 0.479861 \\
79 & 0.000496 & 0.039250 & 4.998276 & 1.143914 & 0.486679 \\
83 & 0.000073 & 0.006119 & 3.033136 & 0.686409 & 0.459221 \\
89 & 0.000349 & 0.031120 & 4.164090 & 0.927696 & 0.469899 \\
97 & 0.000171 & 0.016587 & 4.891240 & 1.069191 & 0.473429 \\
101 & 0.000012 & 0.001283 & 5.297012 & 1.147751 & 0.475323 \\
103 & 0.000032 & 0.003301 & 5.144339 & 1.109954 & 0.472822 \\
107 & 0.000030 & 0.003234 & 5.458274 & 1.168087 & 0.473907 \\
109 & 0.000025 & 0.002756 & 6.906638 & 1.472207 & 0.486372 \\
113 & 0.000024 & 0.002809 & 4.021730 & 0.850729 & 0.458353 \\
127 & 0.005911 & 0.750763 & 5.088599 & 1.050454 & 0.468785 \\
131 & 0.000029 & 0.003827 & 2.836826 & 0.581889 & 0.444355 \\
137 & 0.000034 & 0.004791 & 4.937000 & 1.003459 & 0.458862 \\
139 & 0.000079 & 0.011060 & 5.889168 & 1.193474 & 0.465287 \\
149 & 0.000008 & 0.001234 & 5.983424 & 1.195741 & 0.462998 \\
\hline
\end{tabular}

where, using the modified Lemma 3.5 of [34,

(6.4) $\varepsilon_{1}<\frac{q}{x}\left(\frac{\log q \log x}{\log 2}+\frac{q \log q}{4}+15 \log ^{2} q+56 \log q+12\right)<10^{-300} X e^{-X}$.

To estimate the sums over $\rho$, let

$$
R(T)=C_{1} \log (q T)+C_{2}, \quad \phi_{n}(t)=t^{-n-1} \exp \left\{-\frac{\log x}{R \log (q t)}\right\} .
$$


By [34, Lemma 3.7], for each $\chi \neq \chi_{0}$,

$$
\sum_{\substack{\rho:|\gamma| \leqslant H \\ \rho \neq \beta_{0}}} \frac{x^{\beta-1}}{|\rho|}<\varepsilon_{2}+\varepsilon_{3}+\varepsilon_{4}
$$

where, by (6.1),

$$
\begin{aligned}
\varepsilon_{2} & =\frac{1}{2 \sqrt{x}}\left(\frac{\lambda L^{2}}{\pi}+\frac{2+\alpha}{\pi} L+\frac{R(H)}{H}+2 R(1)+C_{1}\right)+\frac{q L+\alpha L^{2}}{x}<10^{-100} X e^{-X}, \\
\varepsilon_{3} & =\phi_{0}(H) R(H)=\frac{C_{1} X+C_{2}}{H} e^{-X}<0.00016 X e^{-X}, \\
\varepsilon_{4} & =\frac{1}{2} \int_{1}^{H} \phi_{0}(t) \log \left(\frac{q t}{2 \pi}\right) d t<\frac{1}{2} \int_{1}^{H} \phi_{0}(t) \log (q t) d t \\
& =\frac{\log ^{2} x}{2 R^{2}} \int_{(1+\alpha) L}^{(1+\alpha)^{2} L} \frac{e^{-u}}{u^{3}} d u<\frac{\log ^{2} x}{2 R^{2}(1+\alpha)^{3} L^{3}} \int_{(1+\alpha) L}^{\infty} e^{-u} d u=\frac{X e^{-X}}{2} .
\end{aligned}
$$

Therefore,

$$
\varepsilon_{2}+\varepsilon_{3}+\varepsilon_{4}<0.5002 X e^{-X} .
$$

For each $\chi \neq \chi_{0}$, [34, Lemma 3.8] implies that

$$
\sum_{\rho:|\gamma|>H} \frac{x^{\beta-1}}{|\rho(\rho+1)(\rho+2)|}<\varepsilon_{5}+\varepsilon_{6}+\varepsilon_{7},
$$

where

$$
\begin{aligned}
\varepsilon_{5} & =\frac{1}{2 H^{2} \sqrt{x}}\left(\frac{H}{2 \pi}(1+\alpha) L+2 R(H)+\frac{C_{1}}{3}\right)+\frac{4 L}{x H^{2}}<10^{-100} \frac{X e^{-X}}{H^{2}}, \\
\varepsilon_{7} & =R(H) \phi_{2}(H)=\frac{C_{1} X+C_{2}}{H^{3}} e^{-X}<0.00016 \frac{X e^{-X}}{H^{2}} \\
\varepsilon_{6} & =\frac{1}{2} \int_{H}^{\infty} C_{1} \phi_{3}(t)+\phi_{2}(t) \log \left(\frac{q t}{2 \pi}\right) d t<\frac{1}{2} \int_{H}^{\infty} \phi_{2}(t) \log (q t) d t \\
& =\frac{q^{2} \lambda L^{2}}{4} \int_{\sqrt{2}}^{\infty} u e^{-\frac{X}{\sqrt{2}}\left(u+\frac{1}{u}\right)} d u=\frac{q^{2} \lambda L^{2}}{2 \pi} K_{2}(2 \sqrt{2} X, \sqrt{2})
\end{aligned}
$$

where $K_{2}$ is the incomplete Bessel function. By [46, Lemmas 4 and 5],

$$
K_{2}(z, x) \leqslant\left(x+\frac{2}{z}\right)\left(\frac{x^{2}}{z\left(x^{2}-1\right)}\right) e^{-\frac{z}{2}(x+1 / x)} \quad(x>1, z>0),
$$

hence

$$
\varepsilon_{6} \leqslant \frac{q^{2}}{2 \pi}\left(X+\frac{1}{2}\right) e^{-3 X}=\frac{X}{2 \pi}\left(1+\frac{1}{2 X}\right) \frac{e^{-X}}{H^{2}} \leqslant \frac{0.1678 X e^{-X}}{H^{2}} .
$$

Therefore,

$$
\varepsilon_{5}+\varepsilon_{6}+\varepsilon_{7}<\frac{0.168 X e^{-X}}{H^{2}} .
$$

By (6.2),

$$
\frac{\delta}{q-1} \leqslant \frac{2.0003}{q H}=2.0003 e^{-X}<\frac{2.0003}{L} X e^{-X} .
$$


Combining this with estimates (6.3), (6.4), (6.5), (6.6), (6.7) and (6.8), we conclude that

$$
\begin{aligned}
\left|E_{q}(x)\right|< & B(q) \frac{(1+\delta) x^{\beta_{0}}}{(q-1) \beta_{0}}+X e^{-X} x\left[(1+\delta)(0.5002)+10^{-300}+0.168 \frac{A_{2}(\delta)}{H^{2}}\right] \\
& +\frac{\delta x}{q-1} \\
< & B(q) \frac{1.012 x^{\beta_{0}}}{q}+\frac{8}{9} x X e^{-X} .
\end{aligned}
$$

\section{ACKNOWLEDGMENT}

The second author worked on this project during a visit to CWI in Amsterdam in October 2010 as a Beeger lecturer. Both the second and third authors thank H. te Riele for his hospitality at CWI where part of this work was done. The third author would also like to thank Y. Hashimoto and Y. Ihara for kindly sending him [20], respectively [23, and helpful e-mail correspondence. V. Kumar Murty pointed out the relevance of [24] and [41. MPIM-intern Philipp Weiss computed $i_{0}$ and $i_{1}$. The authors thank C. Pomerance for bringing to their attention Erdös' paper [10, they also thank A. Granville for pointing out his paper [15] and W. Bosma and P. Pollack for helpful conversations.

\section{REFERENCES}

[1] George E. Andrews and Bruce C. Berndt, Ramanujan's lost notebook. Part III, Springer, New York, 2012. MR2952081

[2] A. I. Badzyan, The Euler-Kronecker constant, Mat. Zametki 87 (2010), no. 1, 35-47, DOI 10.1134/S0001434610010050 (Russian, with Russian summary); English transl., Math. Notes 87 (2010), no. 1-2, 31-42. MR2730381

[3] Bruce C. Berndt and Ken Ono, Ramanujan's unpublished manuscript on the partition and tau functions with proofs and commentary, Sém. Lothar. Combin. 42 (1999), Art. B42c, 63. The Andrews Festschrift (Maratea, 1998). MR1701582 (2000i:01027)

[4] Yann Bugeaud, Maurice Mignotte, and Samir Siksek, Classical and modular approaches to exponential Diophantine equations. I. Fibonacci and Lucas perfect powers, Ann. of Math. (2) 163 (2006), no. 3, 969-1018, DOI 10.4007/annals.2006.163.969. MR2215137 (2007f:11031)

[5] Harold Davenport, Multiplicative number theory, 3rd ed., Graduate Texts in Mathematics, vol. 74, Springer-Verlag, New York, 2000. Revised and with a preface by Hugh L. Montgomery. MR.1790423 (2001f:11001)

[6] Hubert Delange, Sur des formules de Atle Selberg, Acta Arith. 19 (1971), 105-146. (errata insert) (French). MR0289432 (44 \#6623)

[7] Christopher Deninger, On the analogue of the formula of Chowla and Selberg for real quadratic fields, J. Reine Angew. Math. 351 (1984), 171-191, DOI 10.1515/crll.1984.351.171. MR749681 (86f:11085)

[8] L.E. Dickson, A new extension of Dirichlet's theorem on prime numbers, Messenger of Math., 33 (1904), 155-161.

[9] P.D.T.A. Elliott and H. Halberstam, A conjecture in prime number theory, Symp. Math. 4 (1968-69), 59-72. MR.0276195 (43 \#1943)

[10] P. Erdős, On a problem of G. Golomb, J. Austral. Math. Soc. 2 (1961/1962), 1-8. MR.0123539 (23 \#A864)

[11] FFTW Fast Fourier Transform C Library, available at http://www.fftw.org/.

[12] Greg Fee and Andrew Granville, The prime factors of Wendt's binomial circulant determinant, Math. Comp. 57 (1991), no. 196, 839-848, DOI 10.2307/2938721. MR.1094948 (92f:11183)

[13] Tony Forbes, Prime clusters and Cunningham chains, Math. Comp. 68 (1999), no. 228, 1739-1747, DOI 10.1090/S0025-5718-99-01117-5. MR1651752(99m:11007) 
[14] T. Forbes, Prime k-tuples, http://anthony.d.forbes.googlepages.com/ktuplets.htm

[15] A. Granville, On the size of the first factor of the class number of a cyclotomic field, Invent. Math. 100 (1990), no. 2, 321-338, DOI 10.1007/BF01231189. MR1047137 (91i:11145)

[16] Kevin Ford, The distribution of integers with a divisor in a given interval, Ann. of Math. (2) 168 (2008), no. 2, 367-433, DOI 10.4007/annals.2008.168.367. MR2434882(2009m:11152)

[17] A. Fröhlich and M. J. Taylor, Algebraic number theory, Cambridge Studies in Advanced Mathematics, vol. 27, Cambridge University Press, Cambridge, 1993. MR.1215934 (94d:11078)

[18] H. Halberstam and H.-E. Richert, Sieve methods, Academic Press [A subsidiary of Harcourt Brace Jovanovich, Publishers], London-New York, 1974. London Mathematical Society Monographs, No. 4. MR0424730 (54 \#12689)

[19] G.H. Hardy and J.E. Littlewood, Some problems of a "Partitio Numerorum": III On the expression of a number as a sum of primes, Acta Math. 44 (1922), 1-70.

[20] Yasufumi Hashimoto, Yasuyuki Iijima, Nobushige Kurokawa, and Masato Wakayama, Euler's constants for the Selberg and the Dedekind zeta functions, Bull. Belg. Math. Soc. Simon Stevin 11 (2004), no. 4, 493-516. MR2115723(2006b:11106)

[21] Douglas Hensley and Ian Richards, On the incompatibility of two conjectures concerning primes, Analytic Number Theory (Proc. Sympos. Pure Math., Vol. XXIV, St. Louis Univ., St. Louis, Mo., 1972), Amer. Math. Soc., Providence, R.I., 1973, pp. 123-127. MR0340194 (49 \#4950)

[22] Yasutaka Ihara, On the Euler-Kronecker constants of global fields and primes with small norms, Algebraic geometry and number theory, Progr. Math., vol. 253, Birkhäuser Boston, Boston, MA, 2006, pp. 407-451, DOI 10.1007/978-0-8176-4532-8_5. MR2263195 (2007h:11127)

[23] Yasutaka Ihara, The Euler-Kronecker invariants in various families of global fields, Arithmetics, geometry, and coding theory (AGCT 2005), Sémin. Congr., vol. 21, Soc. Math. France, Paris, 2010, pp. 79-102 (English, with English and French summaries). MR2856562 $(2012 \mathrm{k}: 11182)$

[24] Yasutaka Ihara, V. Kumar Murty, and Mahoro Shimura, On the logarithmic derivatives of Dirichlet L-functions at $s=1$, Acta Arith. 137 (2009), no. 3, 253-276, DOI 10.4064/aa1373-6. MR 2496464 (2009m:11135)

[25] E. Landau, Über die Einteilung der positiven ganzen Zahlen in vier Klassen nach der Mindestzahl der zu ihrer additiven Zusammensetzung erforderlichen Quadrate, Arch. der Math. und Phys. (3) 13 (1908), 305-312. (See also his Collected Papers.)

[26] Edmund Landau, Losung des Lehmer'schen Problems, Amer. J. Math. 31 (1909), no. 1, 86-102, DOI 10.2307/2370180 (German). MR1506062

[27] E. Landau, Handbuch der Lehre von der Verteilung der Primzahlen, 3rd ed., Chelsea, New York, 1953.

[28] A. Languasco and A. Zaccagnini, A note on Mertens' formula for arithmetic progressions, J. Number Theory 127 (2007), no. 1, 37-46, DOI 10.1016/j.jnt.2006.12.015. MR2351662 (2009g:11136)

[29] Alessandro Languasco and Alessandro Zaccagnini, On the constant in the Mertens product for arithmetic progressions. I. Identities. part 1, Funct. Approx. Comment. Math. 42 (2010), no. part 1, 17-27, DOI 10.7169/facm/1269437065. MR2640766 (2011b:11127)

[30] A. Languasco and A. Zaccagnini, On the constant in the Mertens product for arithmetic progressions. II. Numerical values, Math. Comp. 78 (2009), no. 265, 315-326, DOI 10.1090/S0025-5718-08-02148-0. MR2448709 (2010g:11164)

[31] Alessandro Languasco and Alessandro Zaccagnini, Computing the Mertens and MeisselMertens constants for sums over arithmetic progressions, Experiment. Math. 19 (2010), no. 3, 279-284, DOI 10.1080/10586458.2010.10390624. With an appendix by Karl K. Norton. MR2743571 (2011j:11247)

[32] Philippe Lebacque, Generalised Mertens and Brauer-Siegel theorems, Acta Arith. 130 (2007), no. 4, 333-350, DOI 10.4064/aa130-4-3. MR2365709(2009f:11073)

[33] E. M. Matveev, An explicit lower bound for a homogeneous rational linear form in logarithms of algebraic numbers. II, Izv. Ross. Akad. Nauk Ser. Mat. 64 (2000), no. 6, 125-180, DOI 10.1070/IM2000v064n06ABEH000314 (Russian, with Russian summary); English transl., Izv. Math. 64 (2000), no. 6, 1217-1269. MR1817252(2002e:11091) 
[34] Kevin S. McCurley, Explicit estimates for the error term in the prime number theorem for arithmetic progressions, Math. Comp. 42 (1984), no. 165, 265-285, DOI 10.2307/2007579. MR.726004 (85e:11065)

[35] H. L. Montgomery and R. C. Vaughan, The large sieve, Mathematika 20 (1973), 119-134. MR0374060 (51 \#10260)

[36] Hugh L. Montgomery and Robert C. Vaughan, Multiplicative number theory. I. Classical theory, Cambridge Studies in Advanced Mathematics, vol. 97, Cambridge University Press, Cambridge, 2007. MR2378655 (2009b:11001)

[37] Pieter Moree, On some claims in Ramanujan's 'unpublished' manuscript on the partition and tau functions, Ramanujan J. 8 (2004), no. 3, 317-330, DOI 10.1007/s11139-004-0142-4. MR2111687(2005h:11214)

[38] Pieter Moree, Chebyshev's bias for composite numbers with restricted prime divisors, Math. Comp. 73 (2004), no. 245, 425-449 (electronic), DOI 10.1090/S0025-5718-03-01536-9. MR2034131 (2005b:11154)

[39] P. Moree, Values of the Euler phi function not divisible by a prescribed odd prime, math.NT/0611509, 2006, unpublished preprint.

[40] Pieter Moree, Counting numbers in multiplicative sets: Landau versus Ramanujan, Math. Newsl. 21 (2011), no. 3, 73-81. MR3012680

[41] V. Kumar Murty, The Euler-Kronecker constant of a cyclotomic field, Ann. Sci. Math. Québec 35 (2011), no. 2, 239-247 (English, with English and French summaries). MR2917834

[42] Władysław Narkiewicz, Elementary and analytic theory of algebraic numbers, 2nd ed., Springer-Verlag, Berlin, 1990. MR1055830 (91h:11107)

[43] OEIS Foundation (2011), The On-Line Encyclopedia of Integer Sequences, http://oeis.org/.

[44] Michael Rosen, A generalization of Mertens' theorem, J. Ramanujan Math. Soc. 14 (1999), no. 1, 1-19. MR1700882 (2000e:11143)

[45] J. Barkley Rosser and Lowell Schoenfeld, Approximate formulas for some functions of prime numbers, Illinois J. Math. 6 (1962), 64-94. MR0137689 (25 \#1139)

[46] J. Barkley Rosser and Lowell Schoenfeld, Sharper bounds for the Chebyshev functions $\theta(x)$ and $\psi(x)$, Math. Comp. 29 (1975), 243-269. Collection of articles dedicated to Derrick Henry Lehmer on the occasion of his seventieth birthday. MR.0457373 (56 \#15581a)

[47] Daniel Shanks, The second-order term in the asymptotic expansion of $B(x)$, Math. Comp. 18 (1964), 75-86. MR0159174 (28 \#2391)

[48] Blair K. Spearman and Kenneth S. Williams, Values of the Euler phi function not divisible by a given odd prime, Ark. Mat. 44 (2006), no. 1, 166-181, DOI 10.1007/s11512-005-0001-6. MR2237219 (2007j:11133)

[49] Gérald Tenenbaum, Introduction to analytic and probabilistic number theory, Cambridge Studies in Advanced Mathematics, vol. 46, Cambridge University Press, Cambridge, 1995. Translated from the second French edition (1995) by C. B. Thomas. MR.1342300(97e:11005b)

[50] M. A. Tsfasman, Asymptotic behaviour of the Euler-Kronecker constant, Algebraic geometry and number theory, Progr. Math., vol. 253, Birkhäuser Boston, Boston, MA, 2006, pp. 453458, DOI 10.1007/978-0-8176-4532-8_6. MR2263196 (2007h:11129)

[51] C.J. de la Vallée-Poussin, Recherches analytiques sur la théorie des nombres premiers I, Ann. Soc. Sci. Bruxelles 20 (1896), 183-256.

[52] Lawrence C. Washington, Introduction to cyclotomic fields, Graduate Texts in Mathematics, vol. 83, Springer-Verlag, New York, 1982. MR718674 (85g:11001)

Department of Mathematics, University of Illinois at Urbana-Champaign, 1409 West

Green Street, Urbana, Illinois 61801, USA

E-mail address: ford@math.uiuc.edu

Fundación Marcos Moshinsky, Unam, Circuito Exterior, C.U., Apdo. Postal 70-543, Mexico D.F. 04510, Mexico

E-mail address: fluca@matmor.unam.mx

Max-Planck-Institut für Mathematik, Vivatsgasse 7, D-53111 Bonn, Germany.

E-mail address: moree@mpim-bonn.mpg.de 